$\mathrm{AD}$

Award Number:

W81XWH-08-1-0211

TITLE :

Role of the XIAP-Copper Axis in Prostate Cancer

PRINCIPAL INVESTIGATOR:

Graham F. Brady, B.S.

CONTRACTING ORGANIZATION:

University of Michigan

Ann Arbor, MI 48109-1274

REPORT DATE :

April 2010

TYPE OF REPORT:

Annual Summary

PREPARED FOR: U.S. Army Medical Research and Materiel Command Fort Detrick, Maryland 21702-5012

DISTRIBUTION STATEMENT:

X Approved for public release; distribution unlimited

The views, opinions and/or findings contained in this report are those of the author(s) and should not be construed as an official Department of the Army position, policy or decision unless so designated by other documentation. 


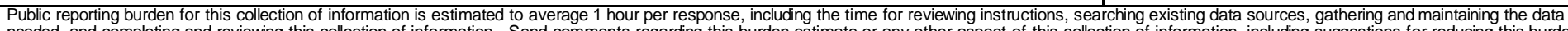

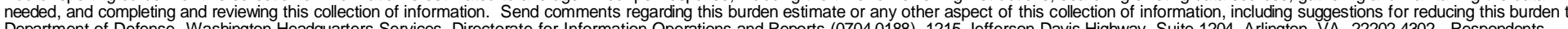

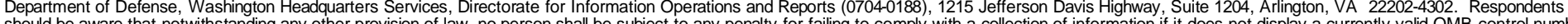
should be aware that notwithstanding any other provision of law, no person shal

\begin{tabular}{l|l|l} 
1. REPORT DATE (DD-MM- $Y Y Y Y)$ & 2. REPORT TYPE & 3. DATES COVERED (FrOm - To)
\end{tabular}

01-04-2010

4. TITLE AND SUBTITLE

Annual Summary

DATES COVERED (From - To)
15 Mar $2008-14$ Mar 2010

Role of the XIAP-Copper Axis in Prostate Cancer

5a. CONTRACT NUMBER

5b. GRANT NUMBER

W81XWH $-08-1-0211$

5c. PROGRAM ELEMENT NUMBER

6. AUTHOR(S)

Graham F. Brady

5d. PROJECT NUMBER

5e. TASK NUMBER

5f. WORK UNIT NUMBER

7. PERFORMING ORGANIZATION NAME(S) AND ADDRESS(ES)

8. PERFORMING ORGANIZATION REPORT NUMBER

University of Michigan

Ann Arbor MI 48109-1274

9. SPONSORING / MONITORING AGENCY NAME(S) AND ADDRESS(ES)

U.S. Army Medical Research and Materiel Command

Fort Detrick, Maryland 21702-5012

10. SPONSOR/MONITOR'S ACRONYM(S)

11. SPONSOR/MONITOR'S REPORT NUMBER(S)

\section{DISTRIBUTION / AVAILABILITY STATEMENT}

Approved for public release; distribution unlimited

\section{SUPPLEMENTARY NOTES}

\section{ABSTRACT}

During the last two years we have been able to identify, confirm, and characterize a novel functional and physical interaction between the X-linked inhibitor of apoptosis (XIAP) and the copper chaperone for superoxide dismutase (CCS). We performed a targeted genetic screen in yeast to identify proteins involved in delivery of copper (Cu) to XIAP. This screen identified CCS as a primary mediator of Cu delivery to XIAP in yeast, and we subsequently determined that CCS delivers $\mathrm{Cu}$ to XIAP in mammalian cells as well. In addition, XIAP targets CCS for ubiquitination through its E3 ubiquitin ligase activity. This ubiquitination event seems to be proteasome-independent and leads to enhancement of CCS activity rather than CCS degradation. Taken together, our results over the first two years of this project have shed substantial light on the interplay between XIAP and copper homeostasis. These studies have the potential to significantly improve our understanding of not only prostate cancer but also other disorders in which XIAP or $\mathrm{Cu}$ metabolism is deregulated.

\section{SUBJECT TERMS}

Apoptosis; Prostate cancer; X-linked inhibitor of apoptosis; Copper metabolism

\section{SECURITY CLASSIFICATION OF:}

a. REPORT

$\mathrm{U}$ b. ABSTRACT $\mathrm{U}$
17. LIMITATION OF ABSTRACT

UU 18. NUMBER
OF PAGES

21 19a. NAME OF RESPONSIBLE PERSON USAMRMC

19b. TELEPHONE NUMBER (include area code) 


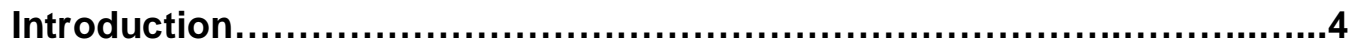

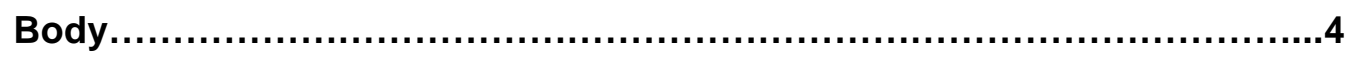

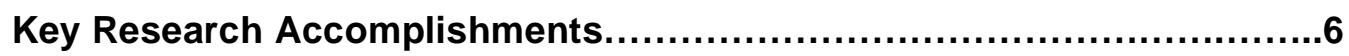

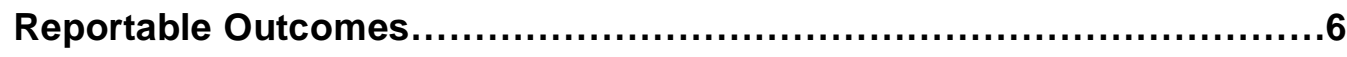

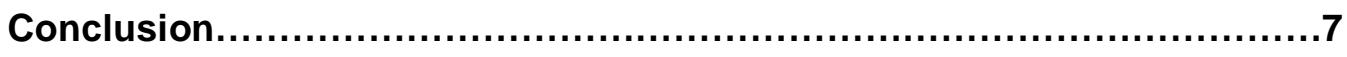

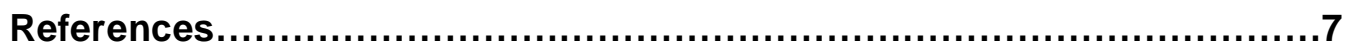

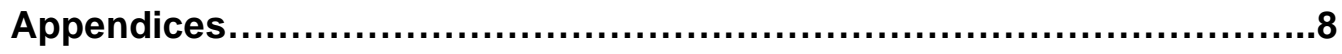




\section{INTRODUCTION}

$\mathrm{X}$-linked inhibitor of apoptosis (XIAP) is commonly deregulated in a number of solid tumor types, including prostate cancer. Similarly, copper $(\mathrm{Cu})$ homeostasis is often altered in malignant tumors and has been implicated in angiogenesis and metastasis, among other processes. XIAP was recently shown to play a role in regulating cellular $\mathrm{Cu}$ metabolism and to be regulated by binding to $\mathrm{Cu}$. This project aims to further delineate the role of XIAP in $\mathrm{Cu}$ metabolism and determine how deregulation of $\mathrm{Cu}$ handling and XIAP function might cooperate in the onset and progression of prostate cancer.

\section{BODY}

I have made substantial progress toward completing the tasks outlined in the original Statement of Work, as outlined below:

AIM 1. How does XIAP affect copper homeostasis in prostate tumors?

These experiments are still in progress. Due to time constraints and technical issues with our in-house atomic absorption spectrometer, we have not completed measurements of copper content from prostate tissue samples as described in Aim 1. During the funds-free third year of this project, we intend to obtain copper measurements from these samples using the more sensitive ICP-MS instrument available on campus in the University of Michigan Department of Geological Sciences.

AIM 2. How is copper delivered to XIAP?

Optimize protocol for monitoring delivery of copper to human XIAP in S. cerevisiae. I was able to establish a reliable protocol for growing yeast transformed with a plasmid encoding human XIAP in $\mathrm{Cu}$-free selective medium. Supplemental $\mathrm{Cu}$ was added to the medium 1-2 hours before cells were harvested for western blotting, which allowed me to accurately monitor delivery of $\mathrm{Cu}$ to XIAP. This objective is complete.

Introduce human XIAP into yeast deletion strains. We selected 16 deletion strains from the same background as our wild-type control (BY4741) for analysis. These 16 strains represented deletions all of the available $\mathrm{Cu}$ chaperones and transporters including the major $\mathrm{Cu}$ importer (Ctr1) and the Wilson/Menkes disease protein homolog (Ccc2). Also included in the 16 strains were several representing putative $\mathrm{Cu}$-binding proteins (eg. Cox11, Cox19, Pet309) and enzymes required for synthesis of the Cu-binding small molecule glutathione (Gsh1, Gsh2). These 16 strains were obtained from Open Biosystems and transformed with the XIAP expression plasmid. This objective is complete.

Assess yeast deletion mutants for delivery of copper to XIAP. After transformation with the XIAP-expressing plasmid, the 16 yeast deletion strains were compared to wildtype yeast in $\mathrm{Cu}$ delivery to XIAP, as determined by western blotting. All mutants tested seemed to deliver $\mathrm{Cu}$ to XIAP with comparable efficiency to wild-type yeast except for the strain lacking the copper chaperone for superoxide dismutase (Ccs). In side-by-side comparisons with wild-type yeast and yeast lacking superoxide dismutase (Sod1), the Ccs 
deletion strain showed a unique and highly reproducible defect in delivery of $\mathrm{Cu}$ to XIAP. This objective is complete.

Confirm findings from yeast in mammalian cells. Embryonic fibroblasts from mice deficient in Ccs, along with littermate controls, were generously provided by Dr. Jonathan Gitlin (Washington University, St. Louis). Fibroblasts lacking Ccs consistently demonstrated a defect in $\mathrm{Cu}$ delivery to endogenous Xiap compared to littermate controls, confirming our findings in yeast with ectopically expressed human XIAP. As additional confirmation, we reduced CCS expression in human embryonic kidney (HEK) $293 \mathrm{~T}$ cells by transient transfection of small interfering RNA (siRNA) oligonucleotides. Reduction in CCS expression produced a corresponding decrease in Cu delivery to XIAP. Conversely, overexpression of human CCS in HEK 293 cells enhanced $\mathrm{Cu}$ delivery to endogenous XIAP. Taken together, our data from yeast and mammalian cells demonstrate a role for $\mathrm{CCS}$ in delivering $\mathrm{Cu}$ to XIAP. This objective is complete.

AIM 3. How does copper availability affect prostate tumor development in the presence and absence of XIAP?

The experiments proposed in Aim 3 were not initiated in the first and second years as we originally planned. We hope it will be possible to complete the animal trial proposed in Aim 3 during the third, funds-free year of the project.

In addition to the progress described above, during the second year of this project we have made significant advances on our progress in year one in characterizing the physical and functional interaction between XIAP and CCS. As described in Aim 2, CCS seems to be the primary mediator of $\mathrm{Cu}$ delivery to XIAP in mammalian cells. The role of CCS in $\mathrm{Cu}$ delivery seems to be direct, as CCS does not have any effect on $\mathrm{Cu}$ export or uptake in mammalian cells (1). In addition, XIAP binds to CCS in HEK 293 cells in a manner that depends on the second domain of CCS. This is the same CCS domain that mediates its interaction with SOD1, suggesting that XIAP might dock with CCS to receive $\mathrm{Cu}$ in a similar manner to SOD1. Interestingly, a point mutant of XIAP that lacks E3 ubiquitin ligase activity (H467A) co-precipitates with CCS more efficiently than wildtype XIAP, suggesting that CCS might be a target for ubiquitination by XIAP. We have found that XIAP does in fact induce ubiquitination of CCS, but it seems to be a proteasome-independent event that does not lead to CCS degradation. Rather, XIAPmediated ubiquitination of CCS seems to positively regulate CCS activity, as CCSmediated metallation of SOD1 is subtly but consistently reduced in lysates from cells in which XIAP expression is reduced by siRNA transfection.

Regulation of CCS by ubiquitination has been previously described, whereby $\mathrm{Cu}$ bound CCS is targeted for proteasome-mediated degradation (2). In order to determine whether XIAP-mediated ubiquitination of $\mathrm{CCS}$ and $\mathrm{Cu}$-induced ubiquitination of CCS represented different pathways, we decided to map the ubiquitinated lysine (Lys) residues of CCS by mass spectrometry. By expressing untagged XIAP with affinity tagged CCS and ubiquitin in HEK 293 cells, we used a two-step purification scheme to obtain ubiquitinated CCS for analysis and identified four lysines on CCS that were conjugated to ubiquitin. Substitution of all four lysines with arginine does not alter binding of CCS to SOD1, suggesting that these lysines are not important to the overall three-dimensional 
structure of CCS. However, absence of the ubiquitinated lysines does seem to impair activation of SOD1, which suggests a functional role for ubiquitination in CCS-mediated metallation of SOD1. Interestingly, XIAP seems to preferentially ubiquitinate CCS at Lys 241, while the $\mathrm{Cu}$-induced ubiquitination of CCS does not exhibit a strong preference for any of the four lysines. The presence of Lys 241 seems to be critical for XIAP to affect activation of SOD1 by CCS, suggesting that ubiquitination of CCS at Lys 241 specifically enhances CCS activity. Further details on this work are included in the attached publication, "Regulation of the copper chaperone CCS by XIAP-mediated ubiquitination."

\section{KEY RESEARCH ACCOMPLISHMENTS}

- Ccs was identified as the primary mediator of $\mathrm{Cu}$ delivery to exogenous human XIAP in yeast.

- Mammalian CCS was determined to mediate $\mathrm{Cu}$ delivery to endogenous XIAP in mouse and human cells.

- A physical interaction between XIAP and CCS was identified and found to depend on the second domain of CCS.

- XIAP was found to ubiquitinate CCS in cells

- CCS protein expression levels were found to be largely independent of XIAP

- XIAP was found to enhance activation of SOD1 by CCS, most likely via CCS ubiquitination.

- The sites of ubiquitination on CCS were mapped by mass spectrometry to four lysine residues.

- XIAP-mediated ubiquitination of CCS was found to occur predominantly at Lys 241.

- Substitution of Lys 241 in CCS was found to alter its activation of SOD1 while leaving its ability to bind to SOD1 intact.

- The effect of XIAP on CCS-mediated SOD1 activation was found to depend on Lys 241, suggesting that ubiquitination by XIAP at this residue specifically enhances $\mathrm{Cu}$ acquisition or $\mathrm{Cu}$ transfer by $\mathrm{CCS}$.

\section{REPORTABLE OUTCOMES}

This work was formally presented in the following settings:

- University of Michigan Medical Scientist Training Program Annual Retreat (August 2009)

- University of Michigan Department of Pathology Defense of Dissertation for the degree Doctor of Philosophy in Molecular and Cellular Pathology (June 2009; degree to be conferred in May 2011)

This work was formally reported in the following publication (attached): 
- Regulation of the copper chaperone CCS by XIAP-mediated ubiquitination. Brady GF, Galbán S, Liu X, Basrur V, Gitlin JD, Elenitoba-Johnson KS, Wilson TE, Duckett CS. Mol Cell Biol. 2010 Apr;30(8):1923-36.

\section{CONCLUSION}

In the first two years of this project, I have made substantial progress toward a better understanding of the role of XIAP in cellular $\mathrm{Cu}$ metabolism. The identification of $\mathrm{CCS}$ as a primary mediator of $\mathrm{Cu}$ delivery to XIAP is intriguing and presents a potential therapeutic approach for targeting XIAP in prostate tumors. $\mathrm{Cu}$-bound XIAP is unstable, yet XIAP levels remain high in many tumors despite elevated intracellular $\mathrm{Cu}$ levels. A drug that could mimic CCS or promote interaction of endogenous CCS with XIAP might prove useful by increasing the amount of $\mathrm{Cu}$ bound to XIAP and thus reducing XIAP levels.

Regulation of SOD1 activation by XIAP through ubiquitination of CCS is surprising and suggests a potential mechanism by which XIAP might enhance tumor cell survival independently of its well-described function in directly blocking apoptosis, by promoting activation of an enzyme that detoxifies reactive oxygen species. Together, the results of years one and two provide valuable insights into the junction between XIAP and $\mathrm{Cu}$ biology and how deregulation of XIAP might intersect with aberrant $\mathrm{Cu}$ homeostasis in prostate cancer development.

\section{REFERENCES}

1. P. C. Wong et al., Proc Natl Acad Sci U S A 97, 2886 (2000).

2. A. L. Caruano-Yzermans, T. B. Bartnikas, J. D. Gitlin, J Biol Chem 281, 13581 (2006). 


\title{
Regulation of the Copper Chaperone CCS by XIAP-Mediated Ubiquitination ${ }^{\nabla}$
}

\author{
Graham F. Brady, ${ }^{1}$ Stefanie Galbán, ${ }^{1}$ Xuwen Liu, ${ }^{1}$ Venkatesha Basrur, ${ }^{1}$ Jonathan D. Gitlin, ${ }^{3}$ \\ Kojo S. J. Elenitoba-Johnson, ${ }^{1}$ Thomas E. Wilson, ${ }^{1}$ and Colin S. Duckett ${ }^{1,2 *}$ \\ Departments of Pathology ${ }^{1}$ and Internal Medicine, ${ }^{2}$ University of Michigan Medical School, Ann Arbor, Michigan 48109, and \\ Department of Pediatrics, Vanderbilt University School of Medicine, Nashville, Tennessee $37232^{3}$
}

Received 9 July 2009/Returned for modification 12 October 2009/Accepted 27 January 2010

\begin{abstract}
In order to balance the cellular requirements for copper with its toxic properties, an elegant set of mechanisms has evolved to regulate and buffer intracellular copper. The X-linked inhibitor of apoptosis (XIAP) protein was recently identified as a copper-binding protein and regulator of copper homeostasis, although the mechanism by which XIAP binds copper in the cytosol is unclear. Here we describe the identification of the copper chaperone for superoxide dismutase (CCS) as a mediator of copper delivery to XIAP in cells. We also find that CCS is a target of the E3 ubiquitin ligase activity of XIAP, although interestingly, ubiquitination of CCS by XIAP was found to lead to enhancement of its chaperone activity toward its physiologic target, superoxide dismutase 1, rather than proteasomal degradation. Collectively, our results reveal novel links among apoptosis, copper metabolism, and redox regulation through the XIAP-CCS complex.
\end{abstract}

Copper is a required cofactor for critical steps in many biological processes, including aerobic respiration, iron metabolism, pigment formation, peptide amidation, neurotransmitter synthesis, connective tissue development, and protection from reactive oxygen species $(29,39,60)$. Although copper is an essential nutrient, the ability of copper ions to easily exchange electrons makes copper highly toxic, so an elaborate system of transporters, chaperones, and chelators has evolved to control the intracellular and extracellular trafficking of copper. Thus, defects in copper uptake or export, at either the cellular or the organismal level, result in pathological copper deficiency or accumulation, respectively.

The importance of copper in mammalian biology is illustrated by the diseases caused by mutations in the genes that encode the copper-transporting ATPases ATP7A and ATP7B. Menkes disease is caused by mutations in the gene that encodes ATP7A, which is essential to bring copper from the digestive tract to other organs. Loss-of-function mutations in $A T P 7 A$ result in severe copper deficiency in all organs but the intestine and kidney, leading to musculoskeletal defects, vascular abnormalities, neurodegeneration, and usually death within the first decade of life (38). Conversely, the copper toxicosis syndrome Wilson disease is caused by mutations in the gene encoding ATP7B, which is highly similar to ATP7A but differs in its intracellular trafficking patterns and tissue distribution (35). Patients with Wilson disease accumulate copper first in the liver and later in other organs, which eventually leads to liver cirrhosis and damage to other organs if copper levels are not reduced therapeutically $(8,23)$.

A number of other copper accumulation disorders have also been described, although their genetic and biochemical mechanisms are generally less well understood. One of the less-

\footnotetext{
* Corresponding author. Mailing address: BSRB Room 2057, 109 Zina Pitcher Place, Ann Arbor, MI 48109-2200. Phone: (734) 6156414. Fax: (734) 763-2162. E-mail: colind@umich.edu.

${ }^{\nabla}$ Published ahead of print on 12 February 2010.
}

characterized disorders of copper accumulation occurs in a subset of Bedlington terriers that lack a functional Commd1 gene (63). COMMD1 is an $\sim 20-\mathrm{kDa}$ protein that has been implicated in a wide variety of pathways, including nuclear factor $\kappa \mathrm{B}$ signaling, response to hypoxia, sodium regulation, and copper homeostasis $(7,17,40,62)$. At least in canines, COMMD1 is required for proper hepatic copper excretion, and it can physically interact with ATP7B, suggesting a common mechanism for human Wilson disease and canine Commd1 deficiency through ATP7B-mediated copper export $(18,58)$.

COMMD1 was independently identified as an interacting partner of the X-linked inhibitor of apoptosis (XIAP) (11). Mammalian inhibitors of apoptosis (IAPs) were originally identified as homologs of a baculovirus IAP and, as the name suggests, were thought to be primarily involved in the regulation of apoptosis $(20,36,61)$. Since their initial characterization, it has become clear that IAPs regulate a wide variety of cellular processes, including mitosis, receptor-mediated signaling pathways, and copper metabolism $(45,55)$. XIAP binds to and ubiquitinates COMMD1 in cells, targeting it for degradation by the proteasome (11). Through this mechanism, XIAP is thought to raise intracellular copper through degradation of a key copper export protein.

Surprisingly, while XIAP regulates copper homeostasis through its interaction with COMMD1, XIAP is in turn regulated by intracellular copper levels. Elevated intracellular copper leads to direct binding of copper to cysteine residues within XIAP, resulting in a distinct conformational change (42). This conformational change leads to an altered electrophoretic mobility of XIAP even under denaturing, reducing conditions, and more importantly, it decreases the stability of XIAP and impairs its ability to inhibit caspases. Thus, XIAP seems to participate in a regulatory loop, promoting its own degradation by raising intracellular copper levels. However, the mechanism by which XIAP binds to copper in the cell remains unclear. Because of its toxicity, intracellular copper is tightly controlled so 
that free copper is unavailable even when total copper is elevated (49). Given that copper-dependent proteins require specific copper chaperones to deliver copper to them, we hypothesized that a chaperone protein might be necessary to mediate copper delivery to XIAP as well.

Through a yeast genetic screen designed to identify candidate proteins involved in delivering copper to XIAP, we identified the copper chaperone for superoxide dismutase (SOD), CCS, as an XIAP-interacting protein. We find that CCS is important for copper delivery to XIAP in mammalian cells and furthermore that CCS is a target for ubiquitination through the E3 ubiquitin ligase activity of XIAP. Surprisingly, ubiquitination of CCS by XIAP seems to be proteasome independent and, rather than triggering degradation of CCS, enhances its ability to deliver copper to its physiologic target, SOD1.

\section{MATERIALS AND METHODS}

Plasmids, oligonucleotides, and transfections. pEBB, pEBG, pEBB-XIAP, and all pEBB-derived XIAP expression plasmids have been described previously $(11,19,34,42)$. For production of lentiviruses, a cassette containing the histone $\mathrm{H} 1$ promoter and a sequence directing the expression of an RNA hairpin targeting either XIAP or green fluorescent protein (GFP) was cloned into the FG12 vector, kindly provided by David Baltimore (48). Human ubiquitin with an amino-terminal histidine tag (pEBB-His ${ }_{6}$-Ubiquitin) and an expression vector with a carboxy-terminal biotinylation sequence tag (pEBB-cTB) were generously provided by Gabriel Maine and Ezra Burstein (University of Texas-Southwestern, Dallas). pEBB-CCS-FLAG was generously provided by Prim de Bie and Leo Klomp (University Medical Center Utrecht, Utrecht, The Netherlands) and was used to generate untagged pEBB-CCS, pEBB-CCS-GST, and pEBB-CCS-cTB. CCS deletion constructs were derived by PCR cloning using primers spanning the appropriate domain(s) containing nested restriction sites. Sequences of these and all other primers are available upon request. Mutant forms of CCS were generated using the QuikChange site-directed mutagenesis kit (Stratagene). To generate pEBB-SOD1-HA, the SOD1 coding sequence was PCR amplified from a human T-cell leukemia cDNA library and cloned into pEBB-(carboxy-terminal)HA. The Saccharomyces cerevisiae expression vector pTW437 is a CEN/ URA3 variant of pTW435, which has been described previously (16). Untagged human XIAP was cloned into pTW437 by gap repair cloning in S. cerevisiae using standard protocols. All small interfering RNA (siRNA) oligonucleotides were obtained from Qiagen. For all experiments using siRNA, an oligonucleotide targeting GFP was used as a control. Double-stranded siRNA oligonucleotides were designed to target the sequences 5'-AACTGCAACAGCTGTGGGAAT-3' (CCS coding sequence nucleotides 418 to 438), 5'-AATGGAGGATGAGCAG CTGAA-3' (CCS coding sequence nucleotides 546 to 566), 5'-AAGTGGTAG TCCTGTTTCAGC-3' (XIAP coding sequence nucleotides 111 to 131), and 5'-AAGACCCGCGCCGAGGTGAAG-3' (GFP coding sequence nucleotides 322 to 342 ). The oligonucleotides targeting XIAP and GFP were described previously (11). Sequences of RNA hairpins for stable suppression of XIAP and GFP were the same as those of the oligonucleotides described above. For transfection of siRNA oligonucleotides, $25 \mu \mathrm{M}$ chloroquinone was added to the culture medium 10 to $15 \mathrm{~min}$ before transfection to enhance transfection efficiency, and the medium was changed to chloroquinone-free medium at $6 \mathrm{~h}$ posttransfection. All transfections were performed using a standard calcium phosphate method.

Yeast strains and conditions. $S$. cerevisiae parental strain BY4741 (MATa his $3 \Delta 1$ leu $2 \Delta 0$ met15 00 ura $3 \Delta 0$ ) and the indicated deletion mutants (obtained from Open Biosystems) were transformed with pTW437-XIAP using standard protocols. Transformed yeast strains were cultured in copper-free minimal selective medium, which was prepared with yeast nitrogen base (YNB) without amino acids, with ammonium sulfate, and lacking copper, iron, zinc, and manganese (US Biological). Synthetic medium $(10 \times)$ deficient in only copper was prepared by adding iron sulfate, zinc chloride, and manganese chloride tetrahydrate to a mixture of YNB and uracil-free complete supplement mixture (CSMURA; Qbiogene) to achieve final concentrations of $2 \mathrm{mg} / \mathrm{liter}$ iron, $4 \mathrm{mg} / \mathrm{liter}$ zinc, and $4 \mathrm{mg} /$ liter manganese in the $10 \times \mathrm{YNB} / \mathrm{CSM}$ mixture. The final copperfree synthetic medium consisted of $1 \times \mathrm{YNB} / \mathrm{CSM}$ and $2 \%$ dextrose in sterile water. Yeast cells were grown at $30^{\circ} \mathrm{C}$ in selective copper-free medium overnight with shaking, diluted at least 10 -fold to an optical density at $600 \mathrm{~nm}\left(\mathrm{OD}_{600}\right)$ of $\sim 0.10$, and allowed to grow to an $\mathrm{OD}_{600}$ of $\sim 0.25$ before the addition of copper,
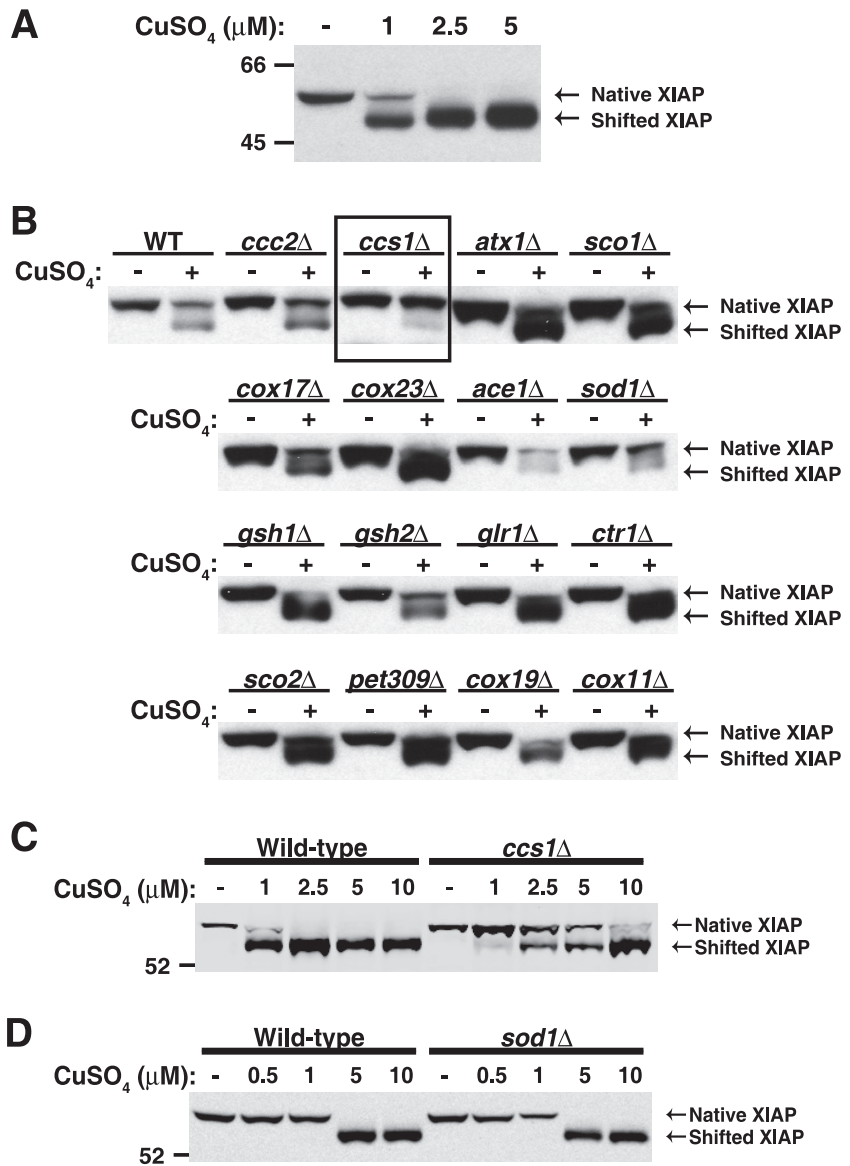

FIG. 1. Ccs1 mediates copper delivery to human XIAP expressed in S. cerevisiae. (A) Wild-type (WT) S. cerevisiae cells were transformed with a plasmid containing the coding sequence of human XIAP, grown in selective medium, and treated with 0 to $5 \mu \mathrm{M} \mathrm{CuSO}_{4}$ for $2 \mathrm{~h}$. Copper delivery to XIAP was determined by SDS-PAGE and Western blot analysis with an antibody directed against human XIAP. Note that copper-bound (shifted) XIAP migrates faster than native XIAP under denaturing, reducing SDS-PAGE conditions. (B) Wild-type yeast and the indicated deletion strains were transformed with human XIAP and treated with 0 or $5 \mu \mathrm{M} \mathrm{CuSO}_{4}$ for $2 \mathrm{~h}$ prior to analysis by Western blotting as for panel A. (C and D) Wild-type yeast cells and yeast cells lacking CCS1 (C) or SOD1 (D) were transformed with a human XIAP expression vector and treated with 0 to $10 \mu \mathrm{M} \mathrm{CuSO}_{4}$ for $1 \mathrm{~h}$. The values to the left of panels $\mathrm{A}, \mathrm{C}$, and $\mathrm{D}$ are molecular sizes in kilodaltons.

additional growth with shaking, and harvesting. Prior to analysis by Western blotting, cells were washed with sterile water, followed by $300 \mathrm{mM} \mathrm{NaOH}$, and then lysed by boiling in NuPAGE lithium dodecyl sulfate sample buffer (Invitrogen) with DL-dithiothreitol (DTT)

Cell lines and culture conditions. Human embryonic kidney (HEK) 293 and $293 \mathrm{~T}$ cells and mouse embryonic fibroblasts (MEFs) were maintained in Dulbecco's modification of Eagle's medium supplemented with $10 \%$ fetal bovine serum and $2 \mathrm{mM}$ L-glutamine at $37^{\circ} \mathrm{C}$ in an atmosphere of $5 \% \mathrm{CO}_{2}$. Generation of Ccs-deficient and Xiap-deficient MEFs, along with wild-type controls, has been previously described $(13,51)$. Stable cell lines were generated by lentiviral infection using FG12 expression vectors containing the relevant hairpin sequences and packaging plasmids as described previously (22). For copper treatments, copper sulfate $\left(\mathrm{CuSO}_{4}\right)$ was obtained from Sigma and copper solutions were freshly prepared for each experiment.

Mice. Xiap-deficient mice were backcrossed in the C57BL/6 strain for at least 12 generations (24). All mice were housed under specific-pathogen-free conditions within the animal care facility at the University of Michigan. All animal 
A
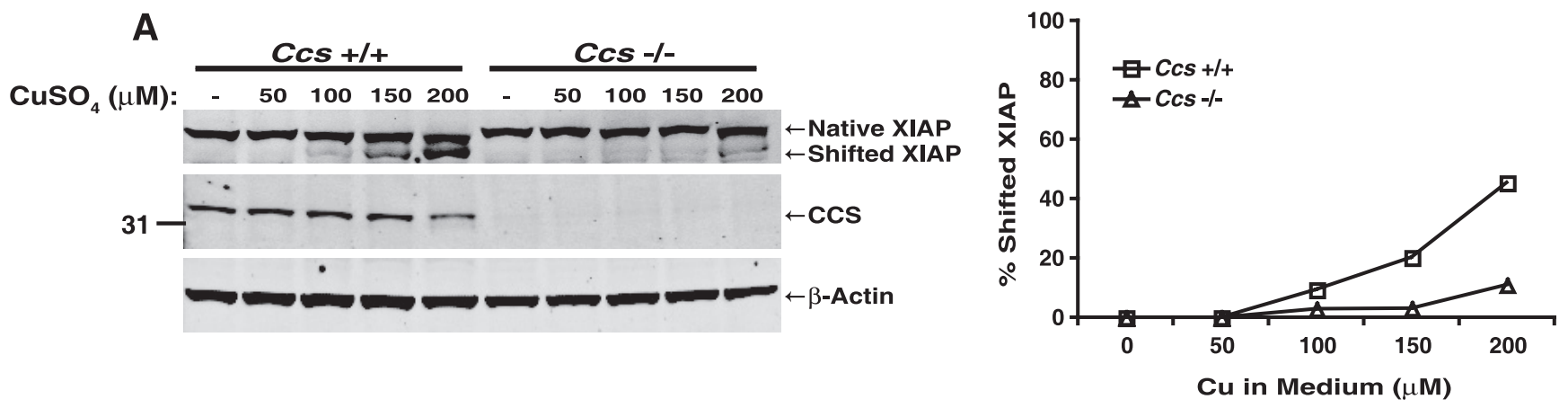

B
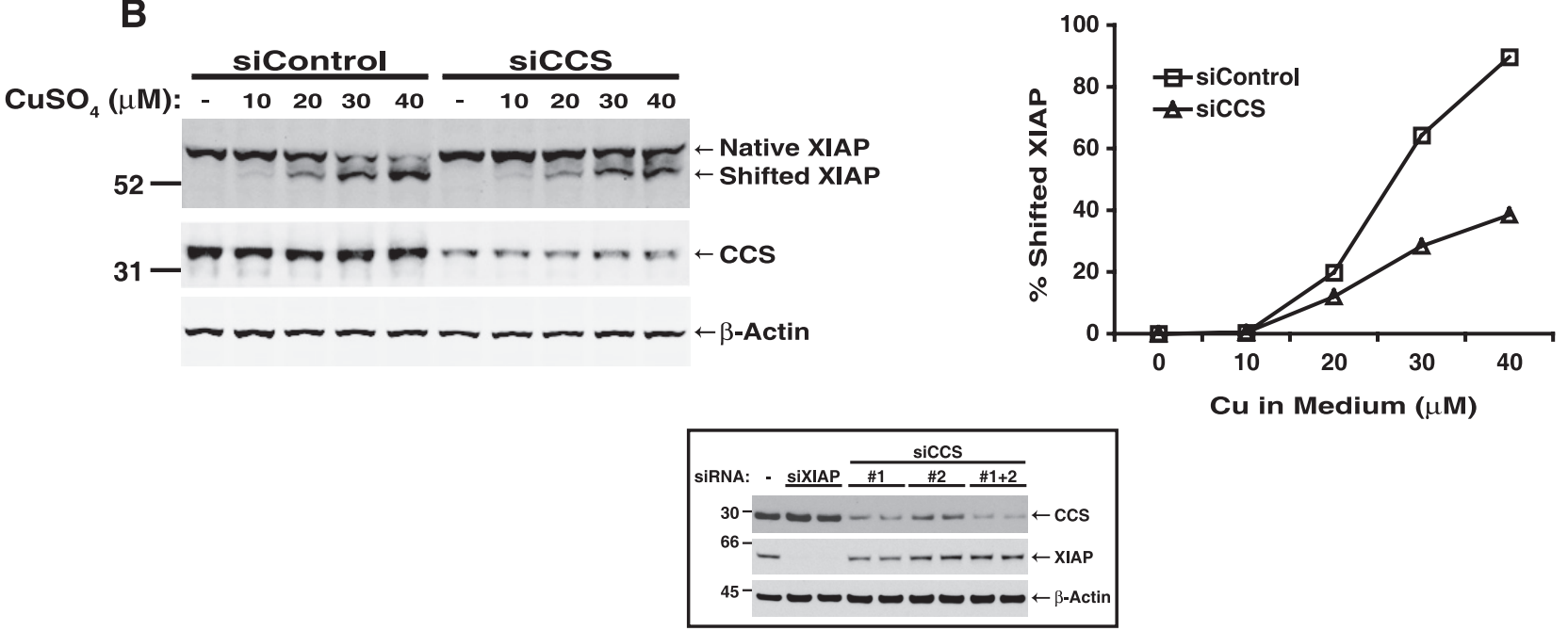

Cu in Medium $(\mu \mathrm{M})$
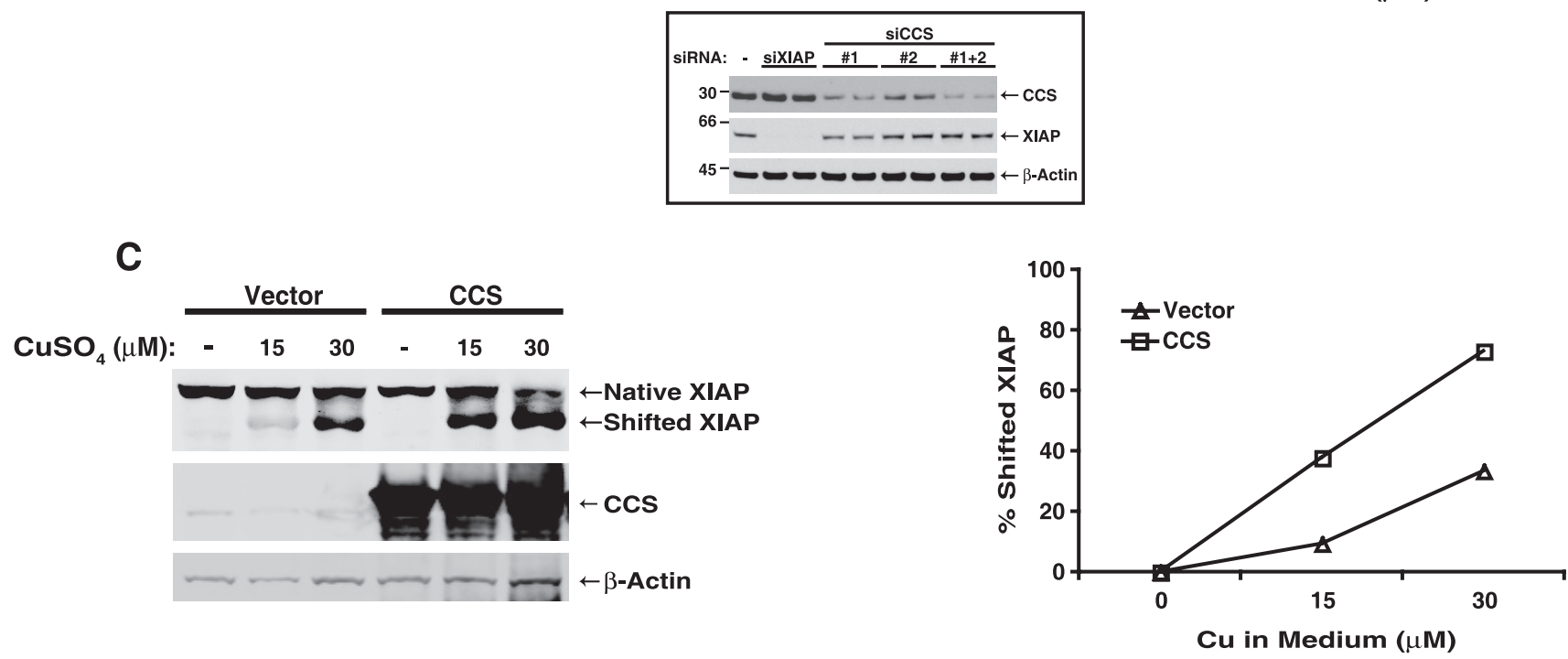

FIG. 2. CCS mediates copper delivery to XIAP in mammalian cells. (A) MEFs derived from either wild-type or Ccs-deficient mice were treated with 0 to $200 \mu \mathrm{M} \mathrm{CuSO}_{4}$ for $48 \mathrm{~h}$. Copper delivery to XIAP was determined by Western blotting and quantitated using Li-Cor Odyssey software as described in Materials and Methods. (B) HEK 293T cells were transfected with a control siRNA oligonucleotide or a combination of two oligonucleotides targeting CCS, incubated for $48 \mathrm{~h}$, and treated with 0 to $40 \mu \mathrm{M} \mathrm{CuSO}_{4}$ for $24 \mathrm{~h}$ prior to lysis and analysis by Western blotting. (Inset) Cells were transfected with siRNA oligonucleotides targeting XIAP or CCS as indicated. Cell lysates were analyzed by Western blotting with antibodies targeting CCS, XIAP, and $\beta$-actin. (C) HEK 293 cells were transfected with a plasmid encoding human CCS or the empty vector and then treated with 0 to $30 \mu \mathrm{M} \mathrm{CuSO}_{4}$ for $48 \mathrm{~h}$ and analyzed by Western blotting as for panels A and B. The quantitative data shown graphically in panels $\mathrm{A}, \mathrm{B}$, and $\mathrm{C}$ were derived from the immunoblots displayed and are representative of at least three independent experiments in each case. The values to the left of the blots in panels $\mathrm{A}$ and $\mathrm{B}$ are molecular sizes in kilodaltons.

experiments were approved by the University of Michigan Committee on the Use and Care of Animals.

Denaturing and native polyacrylamide gel electrophoresis (PAGE) conditions. Unless otherwise noted, cells were harvested with Triton X-100 lysis buffer (1\% Triton X-100, 10\% glycerol, $25 \mathrm{mM}$ HEPES [pH 7.9], $100 \mathrm{mM} \mathrm{NaCl}, 1 \mathrm{mM}$ EDTA, $1 \mathrm{mM}$ DTT, $1 \mathrm{mM}$ phenylmethylsulfonyl fluoride [PMSF], $1 \mathrm{mM}$ sodium orthovanadate, supplemented with protease inhibitors) on ice, and proteins were resolved using 4 to $12 \%$ gradient bis-Tris NuPAGE gels (Invitrogen). Mouse tissues were lysed on ice in radioimmunoprecipitation assay buffer (phosphatebuffered saline containing $1 \%$ Nonidet P- $40,0.5 \%$ sodium deoxycholate, $0.1 \%$ sodium dodecyl sulfate [SDS], $1 \mathrm{mM}$ PMSF, and $1 \mathrm{mM}$ DTT) supplemented with protease inhibitors. For SOD activity assays, lysates were loaded into 4 to $12 \%$ gradient Tris-glycine gels (Invitrogen) and run for $1 \mathrm{~h}$ at room temperature under native conditions. Gels were soaked in deionized water for 30 to $45 \mathrm{~min}$ and then stained for SOD activity according to the method of Beauchamp and Fridovich, with slight modification (4). Briefly, gels were soaked individually in 
A Cu acquisition SOD1 homology Cu delivery

CCS
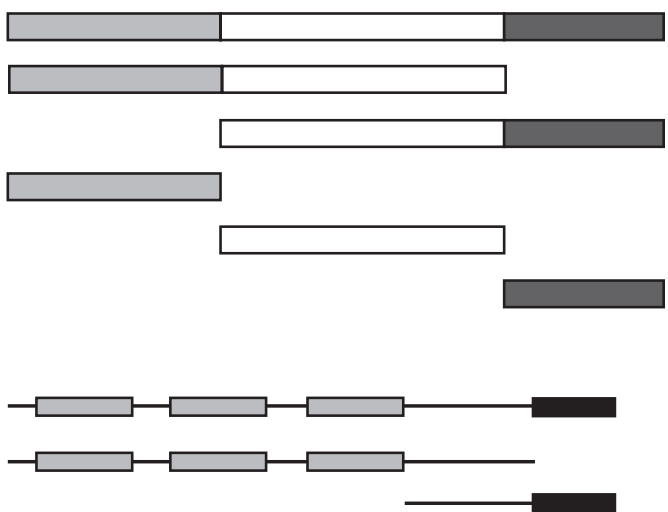

XIAP

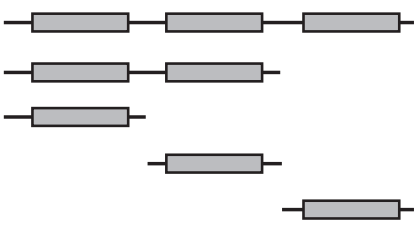

CCS F.L. (1-274)

CCS D1-2 (1-234)

CCS D2-3 (86-274)

CCS D1 (1-85)

CCS D2 (86-234)

CCS D3 (235-274)

XIAP F.L. (1-497)

$\triangle$ RING (1-449)

$\triangle \mathrm{BIR}$ (342-497)

BIR 1-2-3 (1-350)

BIR 1-2 (1-261)

BIR 1 (1-123)

BIR 2 (124-261)

BIR 3 (262-350)

B

INPUT

CCS PD (GSH beads)

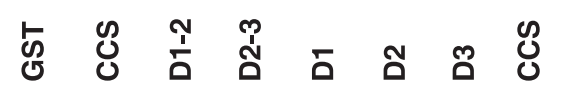

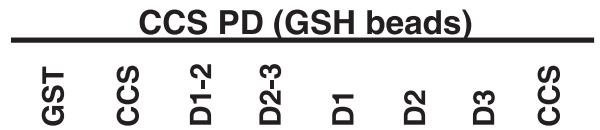

XIAP

$\begin{array}{lllllllllllllllllll}\text { WT } & + & + & + & + & + & + & + & - & + & + & + & + & + & + & + & - \\ \text { H467A } & - & - & - & - & - & - & - & + & - & - & - & - & - & - & - & +\end{array}$
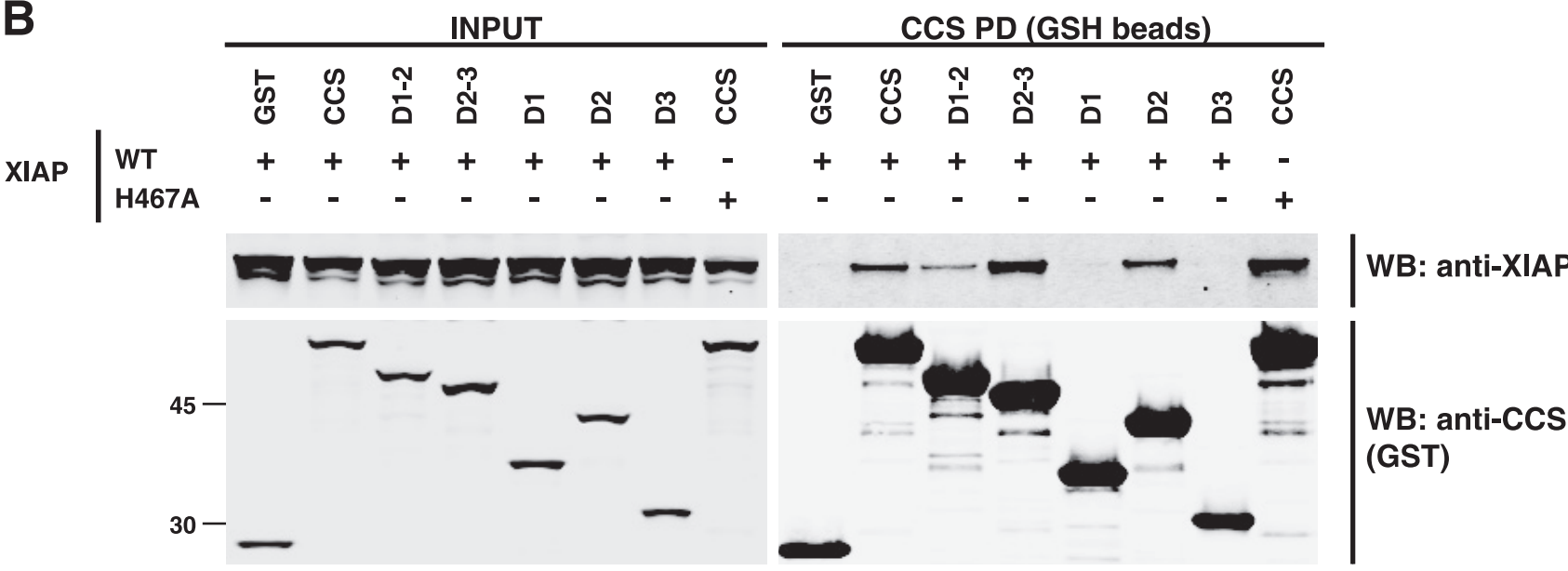

WB: anti-XIAP

C

$$
\begin{aligned}
& \text { CCS-FLAG }
\end{aligned}
$$

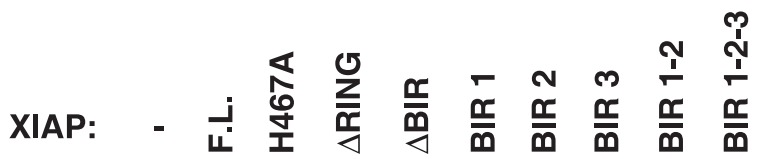

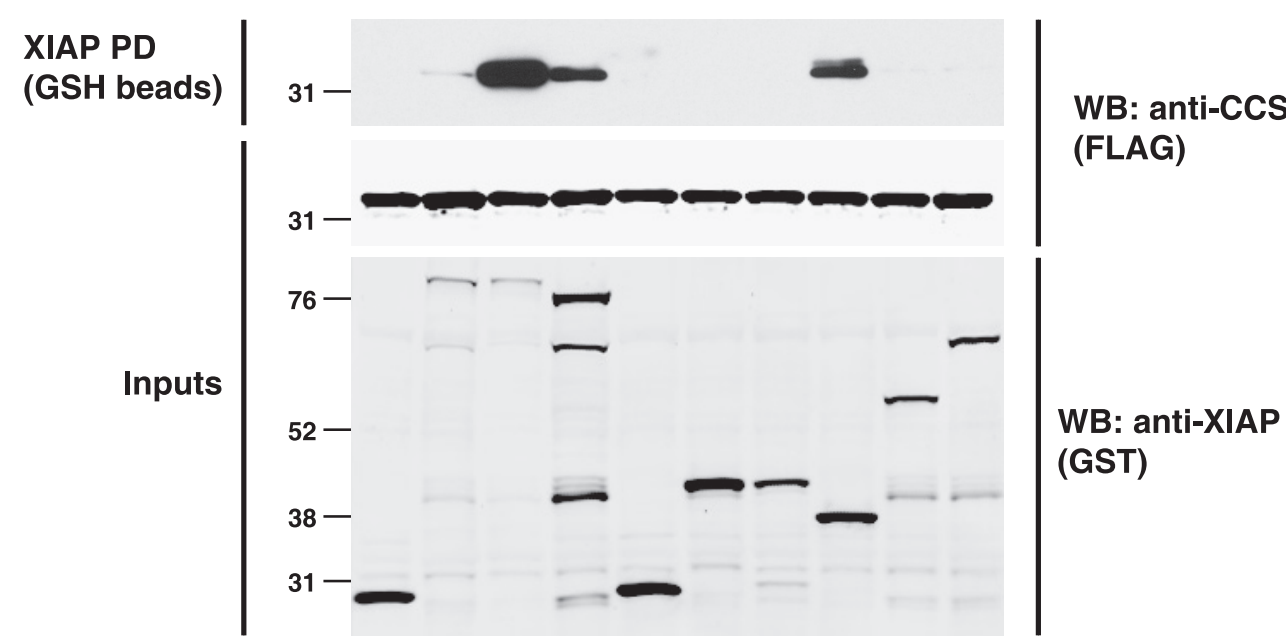


$100 \mathrm{ml} 0.2 \mathrm{mg} / \mathrm{ml}$ nitroblue tetrazolium (NBT; Sigma) for $15 \mathrm{~min}$. After rinsing in water, gels were incubated in $100 \mathrm{ml}$ of $50 \mathrm{mM} \mathrm{KPO}_{4}(\mathrm{pH} 7.8$ ) supplemented with $0.1 \mathrm{mg} / \mathrm{ml}$ riboflavin and $1 \mu \mathrm{l} / \mathrm{ml} N, N, N^{\prime}, N^{\prime}$-tetramethylethylenediamine for $15 \mathrm{~min}$ in the dark. After a brief rinsing, gels were developed by exposure to ambient fluorescent light. SOD gels were imaged on a conventional scanner (Epson), and band intensity was quantitated using ImageJ software (http: //rsbweb.nih.gov/ij/).

Immunoprecipitation, Western blotting conditions, and antibodies. Glutathione $S$-transferase (GST) pulldowns and immunoprecipitations were performed with glutathione (GSH)-Sepharose (GE Healthcare) and anti-hemagglutinin (anti-HA) antibody (Roche), respectively, as described previously (11). For ubiquitination assays, cells were lysed at room temperature under denaturing conditions (8 $\mathrm{M}$ urea, $50 \mathrm{mM}$ Tris [pH 8.0], $300 \mathrm{mM} \mathrm{NaCl}, 50 \mathrm{mM} \mathrm{Na}{ }_{2} \mathrm{HPO}_{4}, 0.5 \%$ NP-40, 1 mM PMSF, supplemented with protease inhibitors) and ubiquitinated material was recovered by rotation with nickel-coated agarose beads (Invitrogen) as described previously $(10,11,40)$. Unless otherwise noted, cells were treated with $40 \mu \mathrm{M}$ MG132 (Sigma) for $4 \mathrm{~h}$ prior to lysis to increase the recovery of ubiquitinated proteins. All Western blot analysis was performed under reducing, denaturing conditions using Invitrogen NuPAGE 4 to $12 \%$ gels as described previously (42). The following primary antibodies were used for Western blot analysis: anti-XIAP (BD Transduction Laboratories), anti-CCS (Santa Cruz), anti- $\beta$-actin (Sigma), anti-FLAG (unconjugated and horseradish peroxidase [HRP; Sigma] conjugated), anti-GST (Santa Cruz), and anti-HA (Covance). In most cases, and in all cases where quantitation of Western blot analysis was performed, blots were scanned using a Li-Cor Odyssey infrared scanner after incubation with the appropriate infrared dye-conjugated secondary antibody (Li-Cor). Band quantitation was performed with Li-Cor Odyssey software (version 2.1) according to the manufacturer's instructions. For Western blots developed with enhanced chemiluminescence (ECL) and film, HRP-conjugated secondary antibodies and ECL were obtained from GE Healthcare and used with Kodak XAR film.

Two-step ubiquitin/biotin pulldown and mass spectrometry (MS). Ubiquitinated and biotinylated CCS was produced in HEK 293 cells by cotransfection of pEBB-CCS-cTB, pEBB-His ${ }_{6}$-Ubiquitin, and pEBB-XIAP and purified via a protocol modified from that developed by Kaiser and colleagues (57). A full description of the two-step purification scheme will be published elsewhere ( $\mathrm{G}$ Maine and E. Burstein, personal communication). Briefly, cells were transfected as described above and treated with exogenous biotin ( $4 \mu \mathrm{M}$; Sigma) and MG132 $(40 \mu \mathrm{M})$ to enhance the recovery of biotinylated, ubiquitinated CCS. As for other His $_{6}$-ubiquitin pulldowns, the cells were lysed with $8 \mathrm{M}$ urea lysis buffer and sonicated. Lysates were rotated sequentially in disposable drip columns (Bio$\mathrm{Rad})$ packed with nickel-coated and then streptavidin-coated agarose beads After the final elution step, proteins were resolved by SDS-PAGE and stained with colloidal Coomassie (Invitrogen) prior to MS analysis at the University of Michigan Department of Pathology Proteomics Facility.

In-gel digestion with trypsin. Protein bands corresponding to unmodified and modified CCS were excised and destained with $30 \%$ methanol for $3 \mathrm{~h}$. Cysteines were reduced and carbamidomethylated by incubating the gel slices with DTT $(10 \mathrm{mM})$, followed by iodoacetamide $(50 \mathrm{mM})$, at room temperature for $30 \mathrm{~min}$ each. Gel slices were crushed, dried using a Vacufuge, and reswollen in $30 \mu \mathrm{l}$ of ammonium bicarbonate buffer containing $500 \mathrm{ng}$ of sequencing grade, modified trypsin (Promega). After 5 min of incubation on ice, $40 \mu \mathrm{l}$ of ammonium bicarbonate buffer was added and digestion was carried out at $37^{\circ} \mathrm{C}$ overnight. An additional $250 \mathrm{ng}$ of trypsin was added $2 \mathrm{~h}$ prior to the extraction of peptides. Peptides were extracted once each with $60 \%$ acetonitrile $/ 0.1 \%$ trifluoroacetic acid (TFA) and acetonitrile/0.1\% TFA. All extracts were pooled and concentrated to a final volume of $30 \mu \mathrm{l}$ using a Vacufuge.

Protein identification by liquid chromatography-tandem MS (MS/MS). Ten microliters of the digest was resolved on a reverse-phase column (Aquasil $\mathrm{C}_{18}$ $75-\mu \mathrm{m}$ inside diameter, 10-cm-long Picofrit column with a $15-\mu \mathrm{m}$ tip; New Objectives, Woburn, MA) using an acetonitrile/ $1 \%$ acetic acid gradient system ( 5 to $60 \%$ acetonitrile over $40 \mathrm{~min}$, followed by a $95 \%$ acetonitrile wash for $5 \mathrm{~min}$ ) at a flow rate of $\sim 300 \mathrm{nl} / \mathrm{min}$. Eluted peptides were directly introduced into an ion-trap mass spectrometer (LTQ-XL; ThermoFisher) equipped with a nanospray source. The mass spectrometer was operated in data-dependent MS/MS mode to acquire a full MS scan (400 to 2,000 m/z), followed by MS/MS on the top five ions from the full MS scan (relative collision energy, $\sim 35 \%$ ). Dynamic exclusion was set to collect two MS/MS spectra on each ion and exclude it for a further $2 \mathrm{~min}$. Raw files were converted to $\mathrm{mzXML}$ format and searched against the human IPI database (v 3.41, 72,254 entries) appended with a decoy (reverse) database using $\mathrm{X}$ !Tandem with the $k$-score plug-in, an open-source search engine developed by the Global Proteome Machine (www.thegpm.orgy). The search parameters included a precursor peptide mass tolerance window of 2 Da and fragment mass tolerance of $0.5 \mathrm{Da}$. Oxidation of methionine (+16 Da), carbamidomethylation of cysteines (+57 Da), and ubiquitination of lysines (+114 Da) were considered variable modifications. The search was restricted to tryptic peptides with only one missed cleavage tolerated. Results of the X!Tandem search were analyzed using the Trans-Proteomic Pipeline containing PeptideProphet (28) and ProteinProphet (43). Proteins fulfilling a ProteinProphet probability score of $>0.9$ (error rate, $<1 \%$ ) were considered bona fide identifications. MS/MS spectra corresponding to peptides that were identified as ubiquitinated with a PeptideProphet score of $>0.95$ were manually verified.

\section{RESULTS}

Copper delivery to XIAP is mediated by the copper chaperone Ccs1 in S. cerevisiae. Given the excess copper-chelating capacity of the cytosolic environment, we reasoned that simple diffusion of copper to XIAP was unlikely to be a primary mechanism for XIAP-copper binding in vivo. We introduced human XIAP into $S$. cerevisiae in order to carry out a targeted genetic screen to identify candidate proteins involved in delivering copper to XIAP. Copper trafficking pathways are remarkably conserved between yeast and mammalian cells, and $S$. cerevisiae has proven to be a useful model for studying mammalian copper homeostasis (50). Consistent with this, we observed that copper was efficiently delivered to human XIAP expressed ectopically in wild-type $S$. cerevisiae (Fig. 1A). Similar to the studies described previously, the copper-bound form of XIAP was distinguished from native XIAP by its faster migration on SDS-PAGE under denaturing, reducing conditions (42).

To identify proteins that might affect copper trafficking to XIAP, we introduced human XIAP into 16 S. cerevisiae deletion strains in which genes encoding individual copper chaperones and transporters or other proteins with putative roles in copper biology had been deleted by homologous recombination. Interestingly, in the majority of the strains tested, copper was delivered to XIAP with efficiency comparable to that in the wild-type strain in the presence of $5 \mu \mathrm{M}$ copper sulfate (Fig. 1B). However, yeast lacking the metal-responsive transcription factor Ace1, the metalloenzyme Sod1, or the copper chaperone Ccs1 (the yeast homolog of human CCS) demonstrated reduced copper delivery to XIAP compared to the wild-type and other strains tested. Ace1 is required for metallothionein gene transcription, and thus, the ace $1 \Delta$ strain is exquisitely sensitive to copper $(9,59)$. As a result, very few viable ace $1 \Delta$ mutant cells were recovered for Western blot analysis after

FIG. 3. CCS domain 2 and XIAP BIR3 mediate the XIAP-CCS interaction. (A) Schematic representations of CCS and XIAP domains and deletion constructs. (B) Full-length CCS-GST and the indicated CCS deletion constructs were coexpressed in HEK 293 cells with either wild-type (WT) XIAP or a point mutant form of XIAP that lacks E3 ubiquitin ligase activity (H467A). CCS was precipitated from whole-cell lysates with GSH beads, and pulldown samples were analyzed by Western blotting. (C) GST-tagged wild-type XIAP and the indicated mutant forms of XIAP were expressed with FLAG-tagged CCS, precipitated from cell lysates with GSH beads, and analyzed by Western blotting (WB). The values to the left of the blots in panels B and $\mathrm{C}$ are molecular sizes in kilodaltons. 
exposure to elevated extracellular copper. Thus, while we cannot exclude a direct role for Ace1 and metallothioneins in delivering copper to XIAP, the apparent defect in the ace1 $\Delta$ mutant strain is likely due to its drastically reduced fitness under conditions of elevated copper. In contrast, the $\operatorname{css} 1 \Delta$ mutant strain did not exhibit a growth defect in the presence of copper and its defect in copper delivery to XIAP was confirmed by direct comparison with the wild-type strain in the presence of 0 to $10 \mu \mathrm{M}$ copper (Fig. 1C). Although some variability in the absolute copper concentration required to produce the electrophoretic mobility shift of XIAP was observed, the difference between the wild-type and $\operatorname{ccs} 1 \Delta$ mutant strains was consistent across multiple experiments, and in all cases, comparisons were made between samples that were treated and analyzed concurrently.

In yeast and mammals, Ccs1/CCS is essential for the delivery of copper to Sod1 but does not participate in copper uptake, export, or copper delivery to other metalloenzymes $(15,66)$. Given that the role of Ccs1 in copper trafficking is limited to Sod1 metallation, its effect on copper delivery to XIAP was likely to be either direct or via Sod1. In contrast to the $\operatorname{css} 1 \Delta$ mutant strain, side-by-side comparison of the wild-type and $\operatorname{sod} 1 \Delta$ mutant strains did not reveal any differences in copper delivery to XIAP (Fig. 1D). Thus, efficient copper delivery to human XIAP expressed ectopically in yeast was found to require endogenous yeast Ccs1, and furthermore, the role of Ccs1 in copper delivery to XIAP was found to be independent of Sod1 activation.

CCS mediates copper delivery to XIAP in mammalian cells. To determine whether CCS might be involved in delivering copper to XIAP in mammalian cells, MEFs derived from Ccsdeficient mice were treated with excess copper and compared to MEFs derived from a littermate control. In agreement with the results observed in $S$. cerevisiae, copper delivery to endogenous XIAP was substantially reduced in MEFs lacking Ccs (Fig. 2A). Similarly, suppression of endogenous CCS expression by transfection of double-stranded siRNA oligonucleotides reduced copper delivery to XIAP compared to control transfection in HEK 293 T cells (Fig. 2B). In order to achieve maximal suppression of CCS, two different oligonucleotides that moderately reduced CCS expression when transfected separately (Fig. 2B, inset) were pooled and transfected together. Conversely, overexpression of CCS lowered the threshold for copper delivery to endogenous XIAP (Fig. 2C). Thus, in mammalian cells, absent or decreased CCS expression reduced, whereas CCS overexpression enhanced, copper delivery to XIAP. Taken together, our data suggest that although it is not absolutely required, CCS plays a major role in the delivery of copper to XIAP.

Of note, greater concentrations of copper were required to produce a mobility shift in XIAP in mammalian than in $S$. cerevisiae cells, which may be due to intrinsic differences in the efficiency of copper uptake between mammalian and yeast cells; alternatively, the difference may be attributable to the greater chelating capacity of the serum-containing medium in which the mammalian cells were grown.

XIAP physically interacts with CCS. To address whether the role of CCS in copper delivery to XIAP might involve a physical interaction between the two proteins, coprecipitation studies were performed with ectopically expressed proteins. CCS or the indicated CCS domain(s) (Fig. 3A) with a carboxy-terminal GST epitope tag was expressed with amino-terminally HAtagged XIAP in HEK 293 cells. Full-length CCS and deletion constructs containing the SOD1-like second domain of CCS efficiently precipitated XIAP from cell lysates, whereas domains 1 and 3 in isolation did not bind to XIAP (Fig. 3B). The observation that domain 2 of CCS was required for interaction with XIAP is interesting, as this domain mediates CCS homodimerization, as well as binding to SOD1 (52). A similar experiment was carried out with full-length CCS and domain deletions of XIAP fused in frame with an amino-terminal GST tag (Fig. 3C). We found that interaction with CCS was mediated by the most carboxy-terminal baculovirus IAP repeat (BIR3) domain of XIAP, as this domain was necessary and sufficient for precipitation of CCS. It is not clear why the BIR1-2-3 construct did not efficiently precipitate with CCS, as BIR3 alone did, but this result was consistent despite adequate expression of the GST-BIR1-2-3 construct. Collectively, these data suggest that the second domain of CCS and the third BIR domain of XIAP are necessary and sufficient to mediate the XIAP-CCS interaction.

Interestingly, a deletion mutant form of XIAP lacking the carboxy-terminal RING domain ( $\triangle$ RING) and full-length XIAP containing a critical RING domain point mutation (H467A), both of which lack E3 ubiquitin ligase activity (67), consistently coprecipitated with CCS more efficiently than did wild-type XIAP (Fig. 3B and C). Based on previous studies of XIAP and its ubiquitination targets $(11,64,65)$, this observation suggested that disruption of the E3 activity of XIAP might capture what would otherwise be a transient XIAP-CCS complex and that CCS might be a target for XIAP-mediated ubiquitination.

XIAP induces CCS ubiquitination. The possibility that XIAP might ubiquitinate CCS was examined by cotransfection of HEK 293 cells with CCS and human ubiquitin carrying an amino-terminal histidine tag in the absence or presence of XIAP. When CCS was expressed alone, low basal levels of CCS ubiquitination were observed, which were increased by cotransfection of XIAP (Fig. 4A). The XIAP-induced increase in CCS ubiquitination required the RING domain of XIAP, consistent with the hypothesis that CCS is a direct ubiquitination target of XIAP. The effect of XIAP transfection was greater than that of treating cells with excess copper, which was previously reported to result in proteasomal degradation of CCS $(6,13)$. However, Ccs levels were found to be unchanged in tissues from Xiap-deficient mice compared to tissues from wild-type mice (Fig. 4B), suggesting that XIAP is not a primary regulator of CCS stability. This is in contrast to the effect of copper, whose levels have been shown to inversely correlate with CCS levels $(5,6,13,47)$. Consistent with previous reports, we found that CCS expression was enhanced in cells treated with the copper(I) chelator bathocuproine disulfonic acid (BCS), and this effect did not require XIAP (Fig. 4C). In addition, XIAP expression, but not copper treatment, induced the accumulation of an apparently monoubiquitinated form of CCS in the whole-cell lysate (Fig. 4A), further suggesting that XIAP-induced ubiquitination and copper-induced ubiquitination of CCS might represent distinct ubiquitination pathways. Indeed, XIAP was able to ubiquitinate a mutant form of CCS that is insensitive to copper $(\mathrm{C} 244 \mathrm{~S} / \mathrm{C} 246 \mathrm{~S})$ with efficiency 


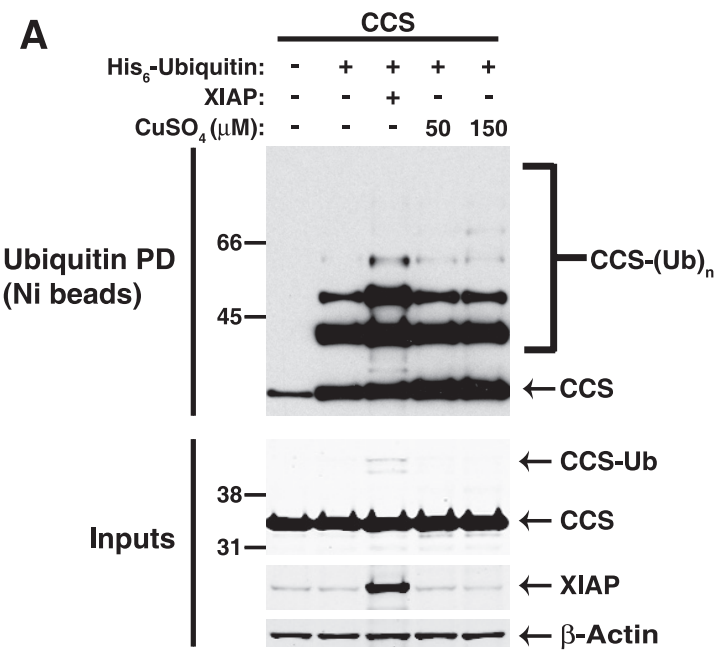

B
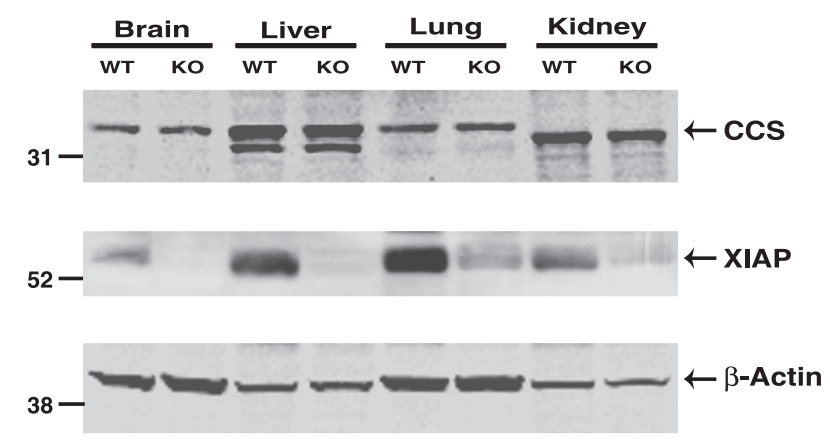

C
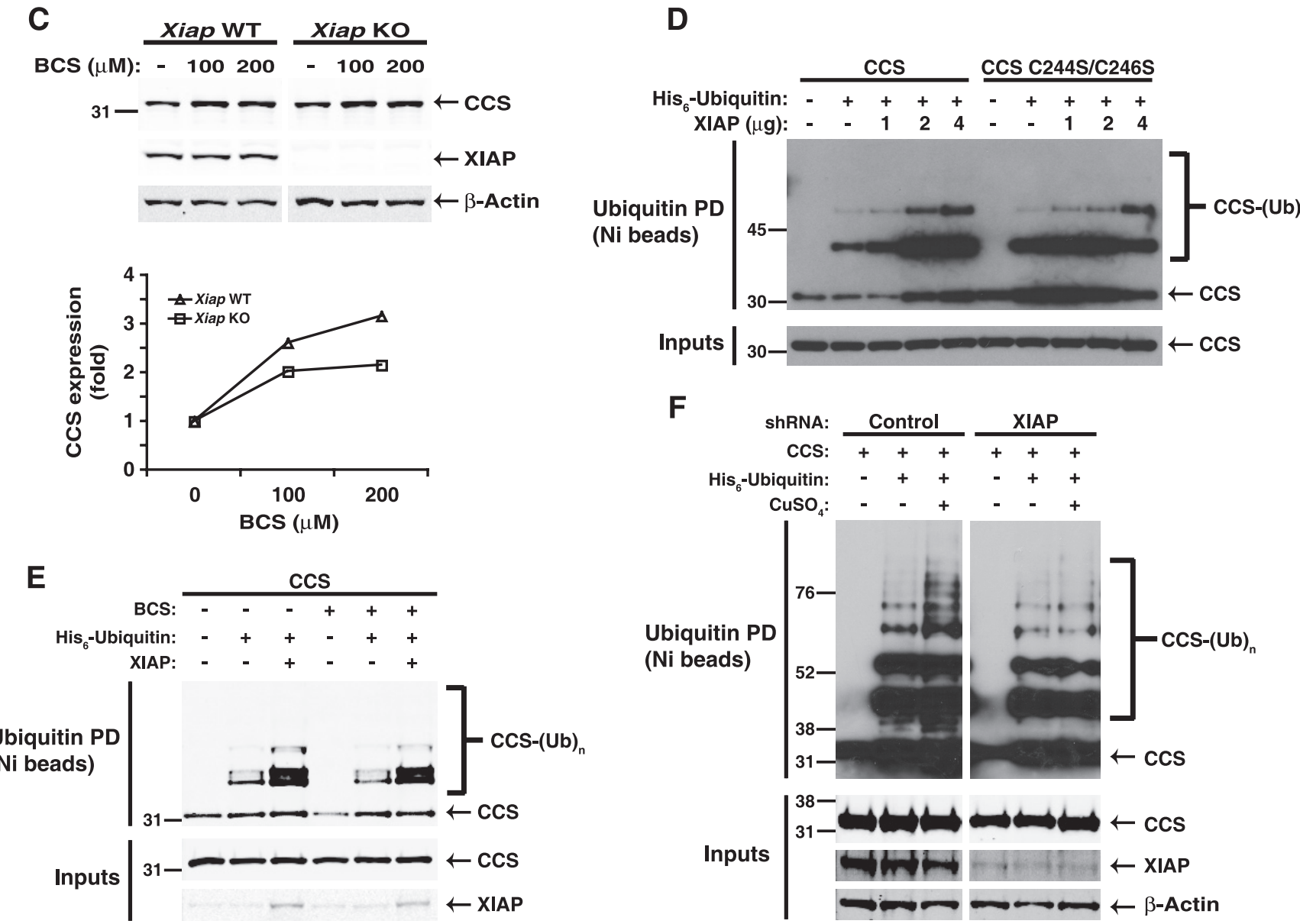

FIG. 4. XIAP ubiquitinates CCS. (A) CCS was coexpressed in HEK 293 cells with histidine-tagged ubiquitin (Ub), and cells were treated by either cotransfecting XIAP or adding copper to the culture medium for $24 \mathrm{~h}$. Ubiquitinated material was recovered from whole-cell lysates by incubation with nickel-coated beads and analyzed by anti-FLAG Western blotting. (B) The indicated tissues were harvested from Xiap-deficient (knockout) and wild-type C57BL/6 mice. Tissue lysates were analyzed by SDS-PAGE and Western blotting with antibodies targeting CCS, XIAP, and $\beta$-actin. WT, wild type; KO, knockout. (C) MEFs derived from Xiap-deficient (KO) and wild-type (WT) mice were treated with the copper chelator BCS for $48 \mathrm{~h}$ and analyzed by SDS-PAGE and Western blotting as indicated. For each condition, CCS protein expression was normalized to $\beta$-actin expression using the Li-Cor Odyssey software as described in Materials and Methods. CCS expression in BCS-treated cells was then quantitated relative to that in untreated cells within each genotype (wild type and Xiap deficient). (D) Wild-type CCS or a copper-resistant mutant form $(\mathrm{C} 244 \mathrm{~S} / \mathrm{C} 246 \mathrm{~S})$ was coexpressed in HEK 293 cells with histidine-tagged ubiquitin (Ub) and increasing amounts of XIAP. Ubiquitinated material was recovered from whole-cell lysates by incubation with nickel-coated beads and analyzed by anti-FLAG Western blotting as for panel A. (E) CCS with a FLAG epitope tag was coexpressed in HEK 293T cells with histidine-tagged ubiquitin (Ub) in the presence or absence of XIAP. Cells were either left untreated or incubated with $150 \mu \mathrm{M} \mathrm{BCS}$ for $24 \mathrm{~h}$ prior to harvesting and analysis by nickel pulldown and Western blotting. (F) HEK 293 cells with stably suppressed XIAP (shXIAP) were derived by lentiviral infection as described in Materials and Methods. The shXIAP cells and control cells were transfected with plasmids encoding human CCS and human ubiquitin and then treated with $50 \mu \mathrm{M} \mathrm{CuSO} \mathrm{for}_{2} 24 \mathrm{~h}$ prior to analysis by nickel pulldown and Western blotting. The values to the left of the blots are molecular sizes in kilodaltons. 

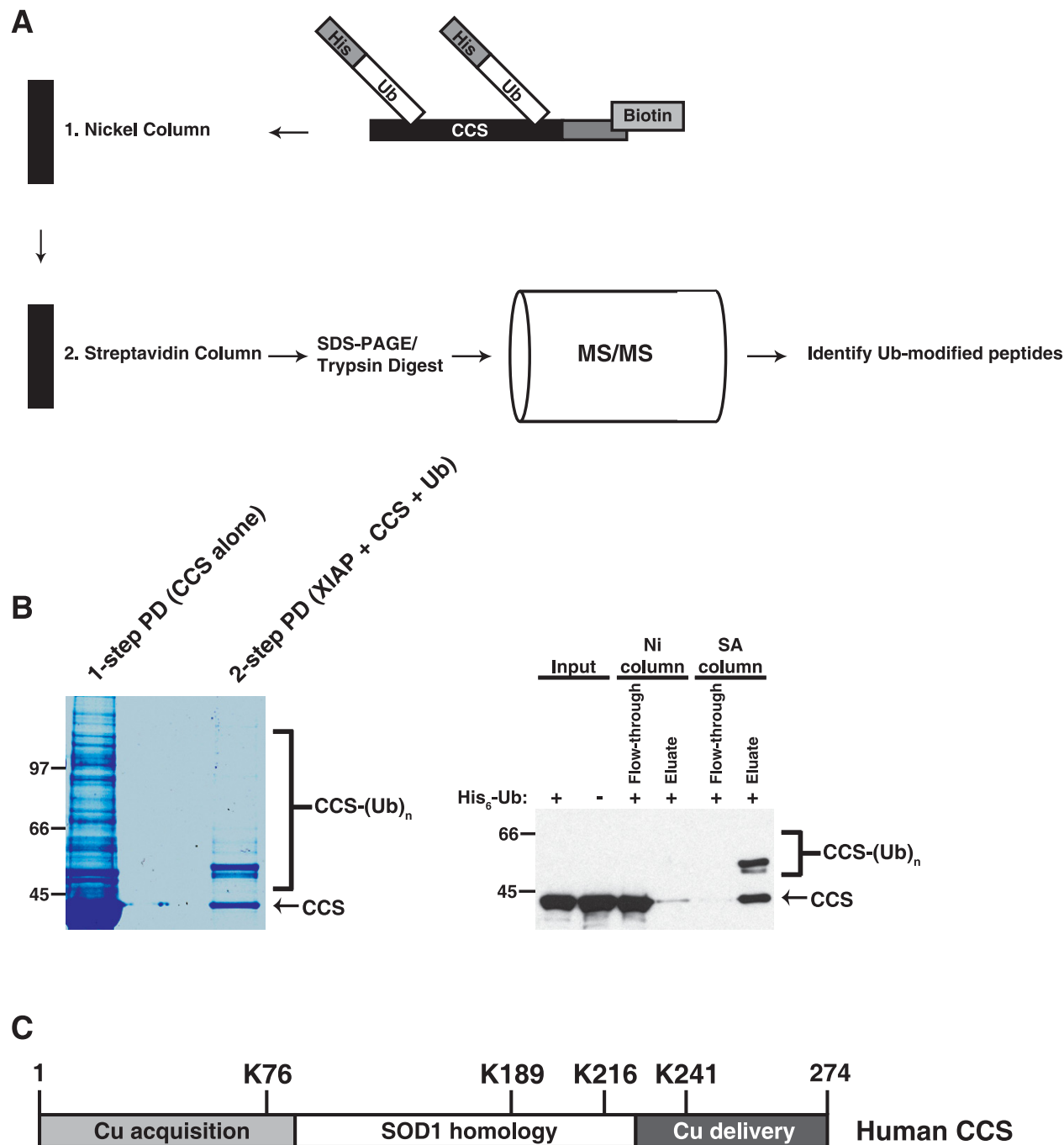

FIG. 5. Identification of ubiquitinated lysine residues in CCS. (A) Ubiquitinated CCS was isolated from HEK 293 cells for MS analysis by a two-step purification scheme using His $_{6}$-ubiquitin and biotinylated CCS. (B) Analysis of eluates from one-step (streptavidin column only) and two-step (nickel column followed by streptavidin column) pulldowns by SDS-PAGE and colloidal Coomassie staining (left panel) and analysis of samples before and after each step of the purification scheme by Western blotting with an antibody directed against CCS (right panel). (C) The four lysines that were identified as ubiquitinated residues by MS are shown in the context of CCS domains 1 to 3 . The values to the left of the blots are molecular sizes in kilodaltons.

comparable to that obtained with wild-type CCS (Fig. 4D) (13). Similarly, treatment with BCS did not alter XIAP-mediated ubiquitination of CCS, suggesting that copper-free CCS may be the primary ubiquitination substrate for XIAP (Fig. 4E). However, both basal ubiquitination and copper-induced ubiquitination of CCS were reduced in HEK 293 cells in which XIAP expression was stably suppressed by a short hairpin RNA (Fig. 4F), suggesting a possible role for XIAP in copperinduced ubiquitination of CCS as well. Taken together, these data suggest that XIAP is an E3 ligase for CCS and that the mechanism and consequences of ubiquitination may be determined by whether or not CCS is bound to copper.

Lysines 76, 189, 216, and 241 of CCS are ubiquitinated in cells. To examine further the mechanistic basis of CCS ubiquitination, the ubiquitinated lysine residues of CCS were mapped by MS. We utilized a two-step affinity purification scheme developed by Burstein and colleagues (Maine and
Burstein, personal communication) to obtain a purified pool of ubiquitinated CCS for analysis (Fig. 5A). Human CCS with a carboxy-terminal biotinylation sequence tag was expressed in HEK 293 cells with histidine-tagged ubiquitin. To increase the total cellular pool of ubiquitinated CCS, untagged XIAP was coexpressed with CCS and ubiquitin and the cells were treated with the proteasome inhibitor MG132 for $4 \mathrm{~h}$ before lysis. A pure preparation of ubiquitinated CCS was obtained by passing the lysate through a nickel column, followed by a streptavidin column, both in the presence of $8 \mathrm{M}$ urea (Fig. 5B). The tagged CCS construct was also expressed separately and purified with the streptavidin column to allow visualization of the unmodified CCS species. Of the seven lysines in human CCS, four were represented in the MS data and all four were ubiquitinated (Fig. 5C). Thus, while only lysines 76, 189, 216, and 241 were observed to be ubiquitinated in this study, it is possible that the remaining three lysines in CCS could be ubiquitinated as well. 


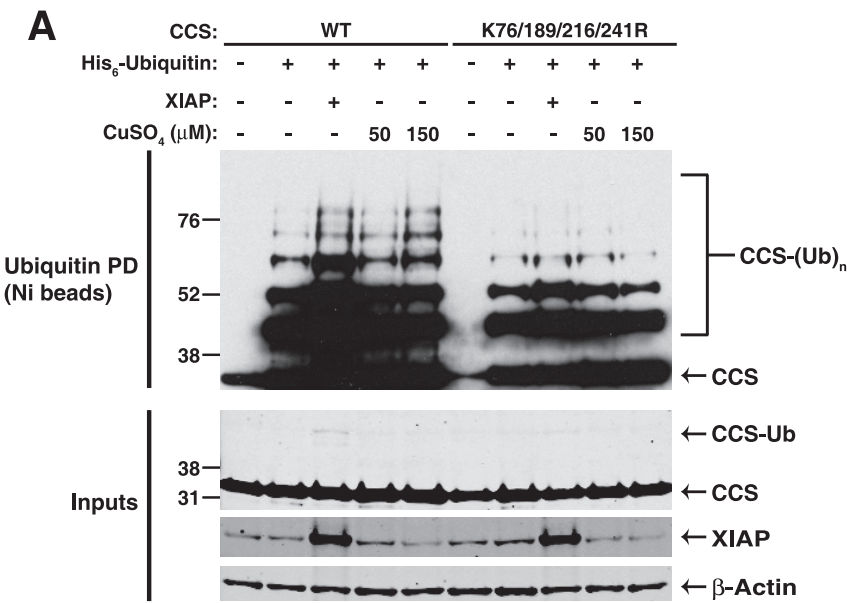

B

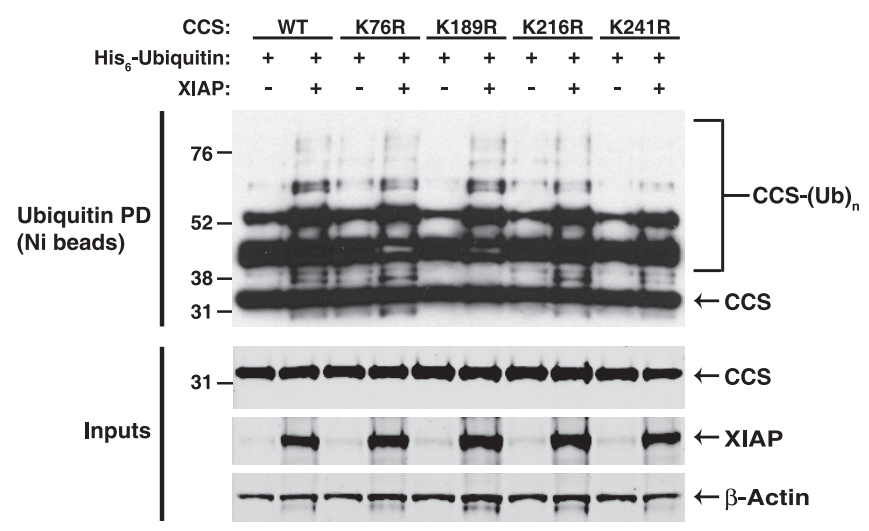

C
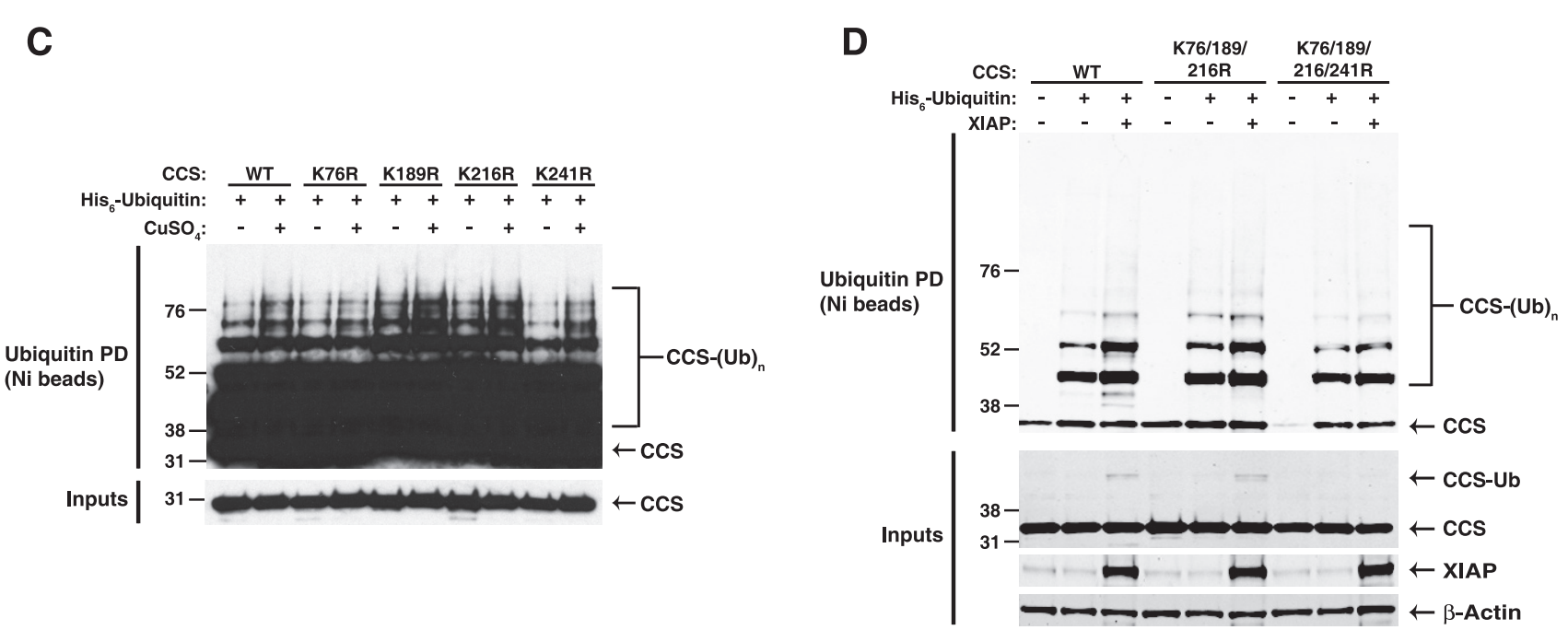

FIG. 6. Preferential ubiquitination of CCS at lysine 241 by XIAP. (A) Wild-type (WT) CCS or a mutant form of CCS in which lysines 76, 189 , 216, and 241 were replaced with arginines was expressed in HEK 293 cells with His $_{6}$-ubiquitin. The effects of XIAP and copper treatment on CCS ubiquitination were determined by Western blot analysis of nickel pulldowns with an anti-FLAG antibody. (B) The effect of XIAP on wild-type CCS and individual lysine mutants was determined as for panel A. (C) Wild-type CCS and the individual lysine mutant forms were coexpressed in HEK 293 cells with histidine-tagged ubiquitin (Ub). Cells were either left untreated or incubated with $150 \mu \mathrm{M}$ copper sulfate for $24 \mathrm{~h}$ prior to harvesting and analysis. (D) The effect of XIAP on the K76/189/216R mutant form of CCS was compared directly to wild-type CCS and the quadruple-lysine mutant form of CCS as for panels A and B. The values to the left of the blots are molecular sizes in kilodaltons.

XIAP preferentially ubiquitinates CCS at lysine 241. In order to investigate the relative contributions of the four identified lysine residues to ubiquitin-dependent regulation of CCS, the four lysine sites were changed to arginines by site-directed mutagenesis. Substitution of arginine residues for all four lysines substantially reduced CCS ubiquitination overall and abrogated XIAP-mediated and copper-induced ubiquitination (Fig. 6A). Analysis of individual CCS lysine mutants in the absence of supplemental copper revealed that replacement of lysine 241 with arginine substantially reduced the ubiquitination of CCS by XIAP compared to that in wild-type CCS and the other individual lysine mutants (Fig. 6B). A similar experiment with copper-treated cells did not reveal a preferred residue for copper-induced CCS ubiquitination (Fig. 6C), supporting the hypothesis that XIAP and copper act on CCS in two distinct ubiquitination pathways. Remarkably, restoration of lysine 241 in the quadruple-lysine mutant form of CCS rescued ubiquitination by XIAP to levels comparable to those of wild-type CCS
(Fig. 6D), suggesting that lysine 241 of CCS is the preferred ubiquitination site for XIAP. Interestingly, lysine 241 is located very close to the copper-binding site in the third domain of CCS, which is required for physiologic metallation of SOD1 by $\operatorname{CCS}(31,52)$, suggesting that XIAP-mediated ubiquitination of CCS might regulate SOD1 activation.

XIAP positively regulates activation of SOD1 by CCS. To determine whether ubiquitination of CCS by XIAP could regulate activation of SOD1, SOD1 and CCS were coexpressed in cells in which XIAP expression was reduced by transient transfection of siRNA. Surprisingly, suppression of endogenous XIAP substantially reduced SOD1 activation, even when CCS was overexpressed (Fig. 7A). This finding suggests that ubiquitination of CCS by XIAP does not inhibit its activation of SOD1 or trigger its proteasomal degradation. Rather, ubiquitination of CCS by XIAP seems to be an activating event, whereby CCS is less efficient at delivering copper to SOD1 in the absence of XIAP. 
A
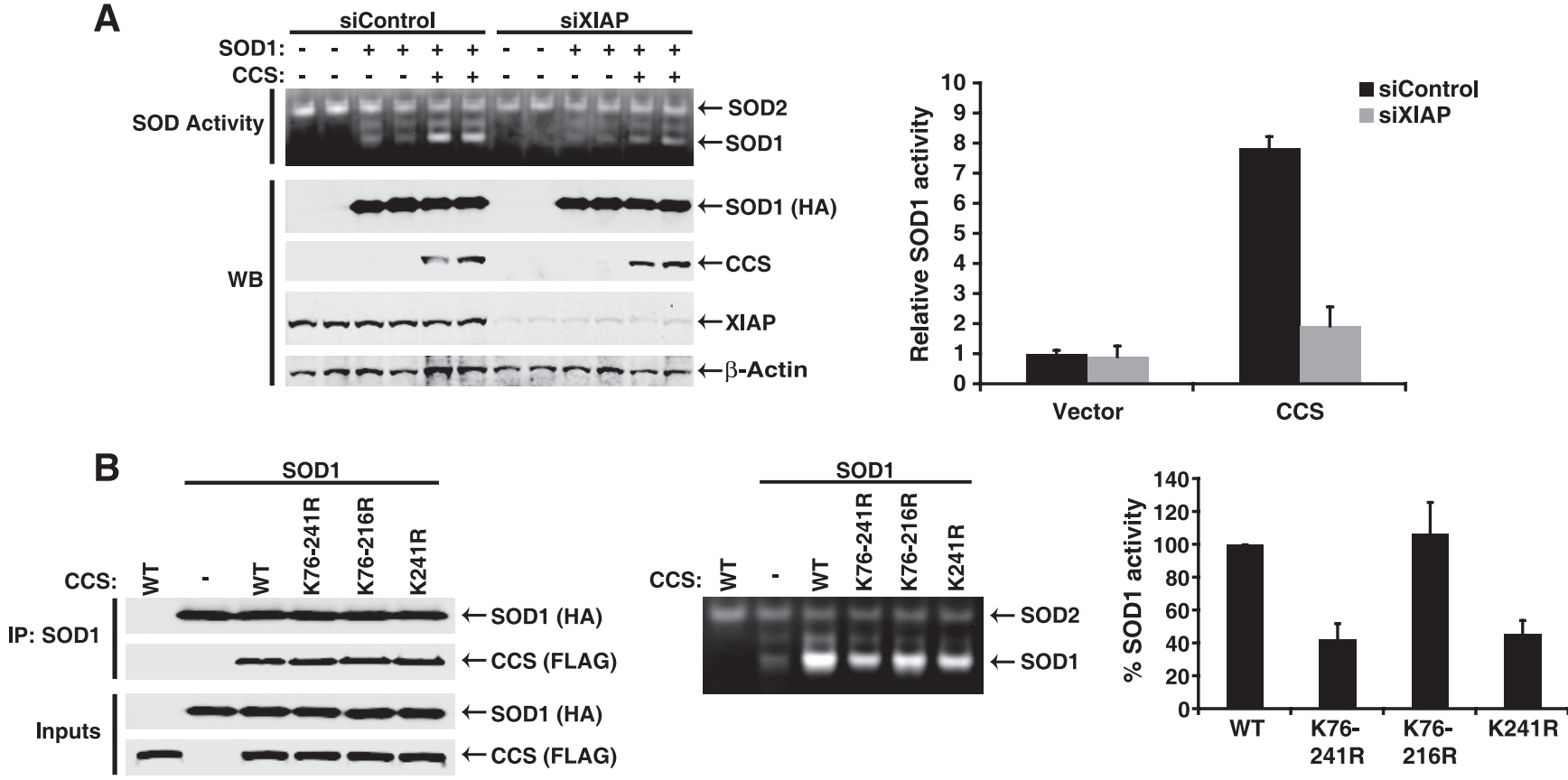

C
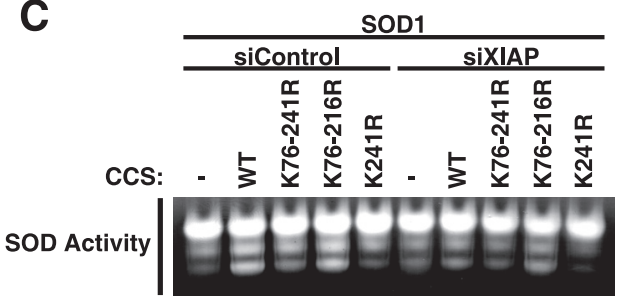

$\leftarrow$ SOD2 $\leftarrow$ SOD1
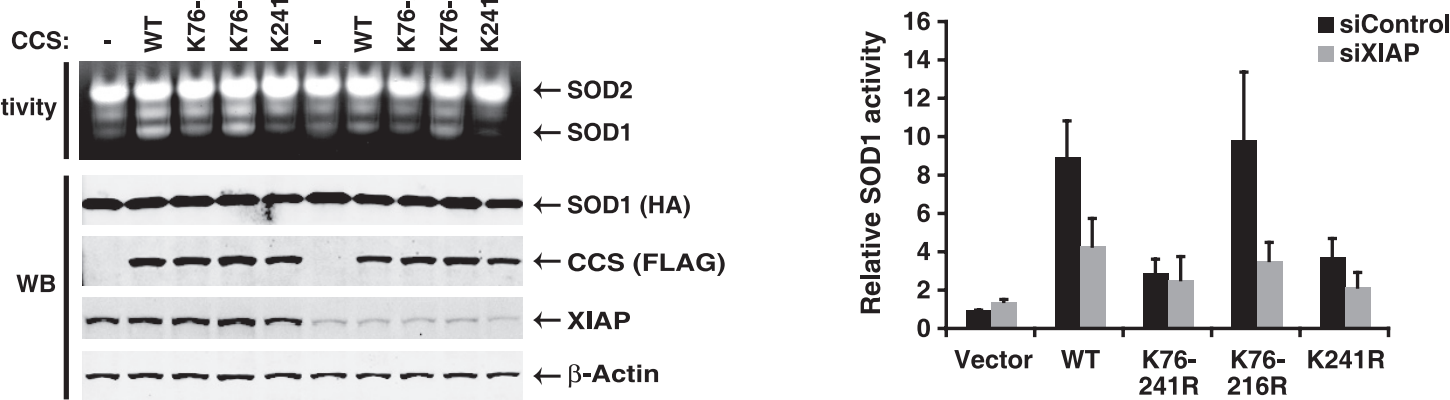

D

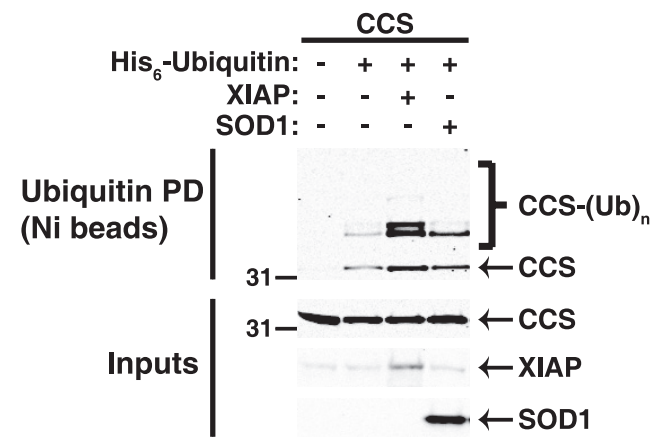

FIG. 7. XIAP-mediated ubiquitination of CCS positively regulates SOD1 activation. (A) Human SOD1 was expressed alone or with human CCS in HEK 293T cells. One day prior to the transfection of plasmid DNA, cells were transfected with a control siRNA oligonucleotide or an oligonucleotide targeting XIAP. Cell lysates were analyzed for SOD1 activity by in-gel NBT staining, and band intensity was quantitated using ImageJ software. Data are presented as the mean \pm the standard deviation of duplicate transfections and are representative of several independent experiments $(n=7)$. WB, Western blotting. (B) Human SOD1 was expressed in HEK 293T cells with wild-type (WT) CCS or the indicated lysine mutant forms of CCS. Two days after transfection, SOD1 was precipitated with an antibody directed against the HA tag and CCS was detected in the immunoprecipitate (IP) with an anti-FLAG antibody (left panel). Whole-cell lysates were analyzed for SOD1 activity by in-gel NBT staining and quantitated as for panel A (middle and right panels). (C) CCS and the indicated mutant forms of CCS were expressed together with SOD1 in cells that had been transfected the previous day with a control siRNA oligonucleotide or an oligonucleotide targeting XIAP, and SOD1 activity was determined as for panels A and B. The data in panels B and C are presented as the mean \pm the standard error of the mean of pooled data from several independent experiments $(n \geq 4)$, with representative SOD gels and Western blot analyses shown. In each case, SOD1 activity was 
Consistent with this notion, the quadruple lysine mutant form of CCS (K76-241R) was substantially impaired in its ability to activate SOD1, although binding to SOD1 was unaffected (Fig. 7B). Replacement of lysine 241 with arginine in wild-type CCS (K241R) reproduced this defect, while restoration of lysine 241 in the quadruple mutant (K76-216R) restored efficient SOD1 activation. Although we cannot exclude an alternative role independent of ubiquitin for lysine 241 in copper delivery to SOD1, the effect of arginine substitution was blunted when XIAP expression was reduced by siRNA transfection (Fig. 7C), suggesting that the role of lysine 241 is as an acceptor site for an XIAP-mediated ubiquitination event that enhances SOD1 activation. In contrast, we could not detect a reciprocal effect of SOD1 on CCS ubiquitination, suggesting that the CCS-SOD1 interaction does not directly regulate CCS ubiquitination (Fig. 7D). Collectively, our data support a model in which XIAP-mediated ubiquitination of CCS, primarily at lysine 241, enhances the ability of CCS to activate SOD1 and SOD1 activation is reduced substantially by the removal of either XIAP or lysine 241 of CCS.

\section{DISCUSSION}

The recent characterization of XIAP as a copper-binding protein provided a novel link between copper homeostasis and apoptosis regulation, but the mechanism and regulation of XIAP-copper binding remain unclear. Here, we used a yeast genetic screen to identify a novel physical and functional interaction between XIAP and the copper chaperone CCS, providing further insight into how intracellular copper and the apoptotic machinery are regulated.

The interaction of XIAP with CCS is an intriguing and potentially important nexus between copper metabolism and apoptosis. We hypothesize that as intracellular copper levels rise, copper-bound CCS accumulates and delivers copper to XIAP. We did not observe any XIAP in the copper-bound state in the absence of supplemental copper, suggesting that XIAP does not compete effectively for copper with SOD1. Based on this observation, it is likely that CCS only delivers copper to XIAP when the cytosolic pool of copper-free SOD1 (apo-SOD1) is essentially depleted. Interestingly, in yeast lacking Sod1, copper delivery to XIAP did not seem to be enhanced compared to that in wild-type yeast, perhaps because of cytosolic copper scavengers or other proteins that compete effectively with XIAP for copper bound to Ccs1. Collectively, the data from yeast and mammalian cells suggest that copper delivery to XIAP is a relatively inefficient process in comparison to SOD1 metallation and requires accumulation of copper-bound CCS above basal levels.

Interestingly, the residual copper-bound XIAP observed under high-copper conditions in $\operatorname{ccs} 1 \Delta$ mutant $S$. cerevisiae (Fig.
1C) and Ccs-deficient MEFs (Fig. 2A) suggests the existence of a CCS-independent pathway for copper delivery to XIAP. This is consistent with previous work by Culotta and colleagues demonstrating CCS-independent metallation of SOD1 via a pathway that requires reduced $\mathrm{GSH}(12,27,32,33)$. It is unclear which protein(s) might be involved in the alternate pathway for copper loading of XIAP. Physical interaction between XIAP and other copper-binding proteins such as CTR1, SOD1, ATP7B, and ATOX1 was not detectable under conditions where a CCS-XIAP interaction was detected (data not shown). It is possible that a nonproteinaceous carrier such as GSH might be involved, as is the case for SOD1. Indeed, XIAP has been shown to bind to copper-bound GSH in vitro (42), suggesting that GSH might be a possible alternative copper source for XIAP. Regardless of the mechanism, our data indicate that CCS-independent copper delivery to XIAP requires substantially higher copper levels than the CCS-dependent pathway, suggesting that CCS-mediated copper delivery is likely to be the predominant pathway for copper loading of XIAP in vivo.

CCS-dependent copper delivery to XIAP may present an opportunity for therapeutic intervention for copper toxicosis disorders or tumors overexpressing XIAP. In the setting of Wilson disease and other copper toxicosis syndromes, hepatic copper levels rise gradually over time, eventually reaching toxic levels and causing local and systemic damage. We hypothesize that an early event in copper toxicosis diseases, before copper levels have risen sufficiently to damage cells directly, may be the accumulation of copper-bound CCS and copper delivery to XIAP. Based on our findings, copper binding to XIAP would be expected to happen when copper levels are only slightly elevated above physiologic levels. Proapoptotic insults due to metabolic toxins and other sources might induce apoptosis in these otherwise healthy cells due to reduced XIAP expression, meaning that liver pathology might begin even when copper accumulation is relatively mild. Similarly, patients undergoing copper-reducing therapy might still be at risk for liver damage via XIAP depletion if hepatic copper levels remain mildly elevated. In this setting, therapeutic targeting of the CCSXIAP interaction to preserve XIAP expression could be beneficial in protecting hepatocytes from indirect copper-mediated toxicity.

Interestingly, elevated XIAP expression has been demonstrated in a variety of tumor types, and targeting of XIAP with antisense oligonucleotides and small molecules has proven effective in preclinical studies $(2,30,41)$. Further study of the XIAP-CCS interaction may provide structural insights that can be used for the rational design of small molecules that can bind copper ions and mimic the XIAP-binding region of CCS to deliver copper to XIAP and trigger its degradation.

Copper delivery to the metalloenzyme SOD1 by CCS is

determined relative to that of the appropriate control (i.e., mock transfection or wild-type CCS) within each experiment before the data were pooled. All of the data obtained for wild-type CCS and the lysine mutant forms of CCS with the control oligonucleotide in panel C were included in the pooled data presented in panel B. (D) CCS with a FLAG epitope tag was coexpressed in HEK 293T cells with histidine-tagged ubiquitin (Ub) in the presence or absence of XIAP or HA-tagged SOD1. After harvesting and lysis, ubiquitinated material was recovered from whole-cell lysates by incubation with nickel-coated beads and CCS ubiquitination was analyzed by anti-FLAG Western blotting. PD, pulldown. The values to the left of the blot in panel D are molecular sizes in kilodaltons. 
Basal copper levels

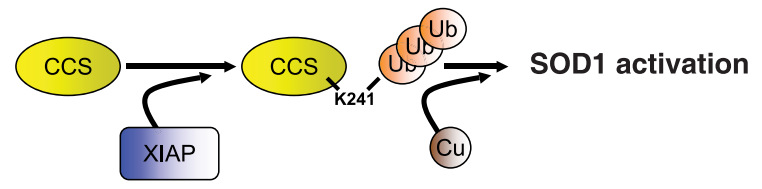

Elevated copper levels

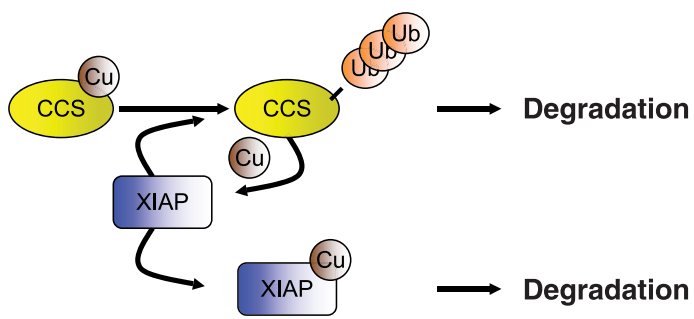

FIG. 8. Proposed model of CCS regulation by distinct ubiquitination pathways. Under basal conditions (top panel), XIAP-mediated ubiquitination of CCS at lysine 241 enhances CCS-mediated activation of SOD1, perhaps by enhancing the ability of CCS to acquire copper. Under conditions of elevated intracellular copper (bottom panel), copper-bound CCS accumulates and transfers copper to XIAP, which is then degraded. Copper-bound CCS is ubiquitinated by XIAP or other cellular E3 ligases and targeted for proteasomal degradation.

essential for SOD1 activation and cellular antioxidant protection (66). Proper metallation of SOD1 is also critical to prevent its misfolding and aggregation, which is thought to be the initiating event in a large subset of familial amyotrophic lateral sclerosis (ALS) (21). Apo-SOD1 is more prone to aggregation than copper-bound SOD1 (holo-SOD1), and several ALS-associated mutations in SOD1 lead to impaired copper binding (25). The role of copper delivery to SOD1 in ALS pathogenesis is unclear, however, as Ccs deficiency does not affect disease progression in mouse models of ALS (56), whereas CCS overexpression leads to accelerated progression in some models but not in others $(53,54)$. Thus, while the CCS-SOD1 interaction is critical for proper intracellular copper homeostasis and redox regulation, the mechanisms by which copper delivery to SOD1 is dynamically regulated and how this process impacts ALS pathogenesis are not clear. Here we describe posttranslational regulation of the CCS-SOD1 interaction through ubiquitination of CCS by XIAP, which could play a regulatory role in maintaining proper folding and activation of SOD1.

Several studies have implicated dynamic posttranslational regulation, including ubiquitination and phosphorylation, of copper chaperones and transporters in maintaining cellular copper homeostasis $(3,13,18,37,44,46)$. A better understanding of how copper trafficking from transporters to chaperones to copper-dependent proteins is dynamically regulated in response to changing cellular requirements will be essential in developing improved therapies for disorders of deregulated copper metabolism.

Posttranslational regulation of CCS by ubiquitination and proteasome-dependent degradation has been described previously $(6,13)$. Our data are consistent with the previous reports of copper-induced CCS degradation and suggest the existence of two distinct pathways of CCS ubiquitination (Fig. 8): (i) ubiquitination of presumably copper-free CCS mediated by XIAP that leads to enhanced copper acquisition and delivery to SOD1 and (ii) ubiquitination of copper-bound CCS by XIAP or another E3 ligase that leads to CCS degradation. Taken together, our data and other previous reports of copper- induced CCS degradation are consistent with a model in which interaction of copper-free CCS and XIAP results in nondegradative ubiquitination of CCS, whereas copper-bound CCS would transfer copper to XIAP and be ubiquitinated and targeted for proteasomal degradation.

The roles of ubiquitin and ubiquitin-like proteins in proteasome-independent regulation of protein function have become increasingly evident in recent years $(1,14,26)$. Addition and removal of monoubiquitin and various types of ubiquitin chains play a critical role in the dynamic regulation of many intracellular processes and signaling events. Here we describe a novel role for the ubiquitin system in the regulation of intracellular redox metabolism through XIAP, CCS, and SOD1. The mechanism by which ubiquitination of CCS might enhance its ability to deliver copper to SOD1 is unclear, but it seems to be independent of CCS-SOD1 binding, suggesting that the ubiquitination status of CCS might instead regulate its acquisition or release of copper to SOD1. Regardless of the precise mechanism by which ubiquitination enhances CCS activity, the effect of lysine 241 ubiquitination on CCS function provides new insights into dynamic regulation of transition metal metabolism and the wide reach of the ubiquitin system in maintaining intracellular homeostasis.

\section{ACKNOWLEDGMENTS}

This work was supported in part by Department of Defense Prostate Cancer Research Program predoctoral award W81XWH-08-1-0211 to G.F.B. and National Institutes of Health grant GM067827 and an American Asthma Foundation award to C.S.D.

We are grateful to Ezra Burstein for providing reagents and protocols, as well as helpful advice and discussions. We also thank Casey Wright and John Wilkinson and members of the Duckett laboratory for insightful discussions and critical reading of the manuscript and David Baltimore, Leo Klomp, and Prim de Bie for plasmids.

\section{REFERENCES}

1. Aguilar, R. C., and B. Wendland. 2003. Ubiquitin: not just for proteasomes anymore. Curr. Opin. Cell Biol. 15:184-190.

2. Amantana, A., C. A. London, P. L. Iversen, and G. R. Devi. 2004. X-linked inhibitor of apoptosis protein inhibition induces apoptosis and enhances chemotherapy sensitivity in human prostate cancer cells. Mol. Cancer Ther. 3:699-707. 
3. Bartee, M. Y., M. Ralle, and S. Lutsenko. 2009. The loop connecting metalbinding domains 3 and 4 of ATP7B is a target of a kinase-mediated phosphorylation. Biochemistry 48:5573-5581.

4. Beauchamp, C., and I. Fridovich. 1971. Superoxide dismutase: improved assays and an assay applicable to acrylamide gels. Anal. Biochem. 44:276287.

5. Bertinato, J., M. Iskandar, and M. R. L’Abbe. 2003. Copper deficiency induces the upregulation of the copper chaperone for $\mathrm{Cu} / \mathrm{Zn}$ superoxide dismutase in weanling male rats. J. Nutr. 133:28-31.

6. Bertinato, J., and M. R. L'Abbe. 2003. Copper modulates the degradation of copper chaperone for $\mathrm{Cu}, \mathrm{Zn}$ superoxide dismutase by the $26 \mathrm{~S}$ proteosome. J. Biol. Chem. 278:35071-35078.

7. Biasio, W., T. Chang, C. J. McIntosh, and F. J. McDonald. 2004. Identification of Murr1 as a regulator of the human $\delta$ epithelial sodium channel. J. Biol. Chem. 279:5429-5434.

8. Brewer, G. J. 2000. Recognition, diagnosis, and management of Wilson's disease. Proc. Soc. Exp. Biol. Med. 223:39-46.

9. Buchman, C., P. Skroch, J. Welch, S. Fogel, and M. Karin. 1989. The CUP2 gene product, regulator of yeast metallothionein expression, is a copperactivated DNA-binding protein. Mol. Cell. Biol. 9:4091-4095.

10. Burstein, E., J. E. Hoberg, A. S. Wilkinson, J. M. Rumble, R. A. Csomos, C. M. Komarck, G. N. Maine, J. C. Wilkinson, M. W. Mayo, and C. S. Duckett. 2005. COMMD proteins, a novel family of structural and functional homologs of MURR1. J. Biol. Chem. 280:22222-22232.

11. Burstein, E., L. Ganesh, R. D. Dick, B. van de Sluis, J. C. Wilkinson, J. Lewis, L. W. J. Klomp, C. Wijmenga, G. J. Brewer, G. J. Nabel, and C. S. Duckett. 2004. A novel role for XIAP in copper homeostasis through regulation of MURR1. EMBO J. 23:244-254.

12. Carroll, M. C., J. B. Girouard, J. L. Ulloa, J. R. Subramaniam, P. C. Wong, J. S. Valentine, and V. C. Culotta. 2004. Mechanisms for activating $\mathrm{Cu}$ - and $\mathrm{Zn}$-containing superoxide dismutase in the absence of the CCS Cu chaperone. Proc. Natl. Acad. Sci. U. S. A. 101:5964-5969.

13. Caruano-Yzermans, A. L., T. B. Bartnikas, and J. D. Gitlin. 2006. Mechanisms of the copper-dependent turnover of the copper chaperone for superoxide dismutase. J. Biol. Chem. 281:13581-13587.

14. Chen, Z. J., and L. J. Sun. 2009. Nonproteolytic functions of ubiquitin in cell signaling. Mol. Cell 33:275-286.

15. Culotta, V. C., L. W. Klomp, J. Strain, R. L. Casareno, B. Krems, and J. D. Gitlin. 1997. The copper chaperone for superoxide dismutase. J. Biol. Chem. 272:23469-23472.

16. Daley, J. M., R. L. Laan, A. Suresh, and T. E. Wilson. 2005. DNA joint dependence of pol $\mathrm{X}$ family polymerase action in nonhomologous end joining. J. Biol. Chem. 280:29030-29037.

17. de Bie, P., B. van de Sluis, E. Burstein, K. J. Duran, R. Berger, C. S. Duckett, C. Wijmenga, and L. W. Klomp. 2006. Characterization of COMMD protein-protein interactions in NF-кB signalling. Biochem. J. 398:63-71.

18. de Bie, P., B. van de Sluis, E. Burstein, P. V. van de Berghe, P. Muller, R. Berger, J. D. Gitlin, C. Wijmenga, and L. W. Klomp. 2007. Distinct Wilson's disease mutations in ATP7B are associated with enhanced binding to COMMD1 and reduced stability of ATP7B. Gastroenterology 133:13161326.

19. Duckett, C. S., F. Li, Y. Wang, K. J. Tomaselli, C. B. Thompson, and R. C. Armstrong. 1998. Human IAP-like protein regulates programmed cell death downstream of Bcl-xL and cytochrome $c$. Mol. Cell. Biol. 18:608-615.

20. Duckett, C. S., V. E. Nava, R. W. Gedrich, R. J. Clem, J. L. Van Dongen, M. C. Gilfillan, H. Shiels, J. M. Hardwick, and C. B. Thompson. 1996. A conserved family of cellular genes related to the baculovirus iap gene and encoding apoptosis inhibitors. EMBO J. 15:2685-2694.

21. Furukawa, Y., and T. V. O'Halloran. 2005. Amyotrophic lateral sclerosis mutations have the greatest destabilizing effect on the apo- and reduced form of SOD1, leading to unfolding and oxidative aggregation. J. Biol. Chem. 280:17266-17274

22. Galbán, S., C. Hwang, J. M. Rumble, K. A. Oetjen, C. W. Wright, A. Boudreault, J. Durkin, J. W. Gillard, J. B. Jaquith, S. J. Morris, and C. S. Duckett. 2009. Cytoprotective effects of IAPs revealed by a small molecule antagonist. Biochem. J. 417:765-771.

23. Gitlin, J. D. 2003. Wilson disease. Gastroenterology 125:1868-1877.

24. Harlin, H., S. B. Reffey, C. S. Duckett, T. Lindsten, and C. B. Thompson. 2001. Characterization of XIAP-deficient mice. Mol. Cell. Biol. 21:3604 3608 .

25. Hayward, L. J., J. A. Rodriguez, J. W. Kim, A. Tiwari, J. J. Goto, D. E. Cabelli, J. S. Valentine, and R. H. J. Brown. 2002. Decreased metallation and activity in subsets of mutant superoxide dismutases associated with familia amyotrophic lateral sclerosis. J. Biol. Chem. 277:15923-15931.

26. Hochstrasser, M. 2009. Origin and function of ubiquitin-like proteins. Nature 458:422-429.

27. Jensen, L. T., and V. C. Culotta. 2005. Activation of CuZn superoxide dismutases from Caenorhabditis elegans does not require the copper chaperone CCS. J. Biol. Chem. 280:41373-41379.

28. Keller, A., A. I. Nesvizhskii, E. Kolker, and R. Aebersold. 2002. Empirical statistical model to estimate the accuracy of peptide identifications made by MS/MS and database search. Anal. Chem. 74:5383-5392.
29. Kim, B. E., T. Nevitt, and D. J. Thiele. 2008. Mechanisms for copper acquisition, distribution and regulation. Nat. Chem. Biol. 4:176-185.

30. LaCasse, E. C., G. G. Cherton-Horvat, K. E. Hewitt, L. J. Jerome, S. J. Morris, E. R. Kandimalla, D. Yu, H. Wang, W. Wang, R. Zhang, S. Agrawal, J. W. Gillard, and J. P. Durkin. 2006. Preclinical characterization of AEG35156/GEM 640, a second-generation antisense oligonucleotide targeting X-linked inhibitor of apoptosis. Clin. Cancer Res. 12:5231-5241.

31. Lamb, A. L., A. S. Torres, T. V. O'Halloran, and A. C. Rosenzweig. 2001. Heterodimeric structure of superoxide dismutase in complex with its metallochaperone. Nat. Struct. Biol. 8:751-755.

32. Leitch, J. M., L. T. Jensen, S. D. Bouldin, C. E. Outten, P. J. Hart, and V. C. Culotta. 2009. Activation of $\mathrm{Cu}, \mathrm{Zn}$-superoxide dismutase in the absence of oxygen and the copper chaperone CCS. J. Biol. Chem. 284:21863-21871.

33. Leitch, J. M., P. J. Yick, and V. C. Culotta. 2009. The right to choose: multiple pathways for activating copper,zinc superoxide dismutase. J. Biol Chem. 284:24679-24683.

34. Lewis, J., E. Burstein, S. Birkey Reffey, S. B. Bratton, A. B. Roberts, and C. S. Duckett. 2004. Uncoupling of the signaling and caspase-inhibitory properties of XIAP. J. Biol. Chem. 279:9023-9029.

35. Linz, R., and S. Lutsenko. 2007. Copper-transporting ATPases ATP7A and ATP7B: cousins, not twins. J. Bioenerg. Biomembr. 39:403-407.

36. Liston, P., N. Roy, K. Tamai, C. Lefebvre, S. Baird, G. Cherton-Horvat, R. Farahani, M. McLean, J.-E. Ikeda, A. MacKenzie, and R. G. Korneluk. 1996 Suppression of apoptosis in mammalian cells by NAIP and a related family of IAP genes. Nature 379:349-353.

37. Liu, J., A. Sitaram, and C. G. Burd. 2007. Regulation of copper-dependent endocytosis and vacuolar degradation of the yeast copper transporter, Ctr1p, by the Rsp5 ubiquitin ligase. Traffic 8:1375-1384.

38. Llanos, R. M., and J. F. Mercer. 2002. The molecular basis of copper homeostasis copper-related disorders. DNA Cell Biol. 21:259-270.

39. Madsen, E., and J. D. Gitlin. 2007. Copper deficiency. Curr. Opin. Gastroenterol. 23:187-192.

40. Maine, G. N., X. Mao, C. M. Komarck, and E. Burstein. 2007. COMMD1 promotes the ubiquitination of NF- $\mathrm{B}$ subunits through a cullin-containing ubiquitin ligase. EMBO J. 26:436-447.

41. McManus, D. C., C. A. Lefebvre, G. Cherton-Horvat, M. St-Jean, E. R. Kandimalla, S. Agrawal, S. J. Morris, J. P. Durkin, and E. C. Lacasse. 2004 Loss of XIAP protein expression by RNAi and antisense approaches sensitizes cancer cells to functionally diverse chemotherapeutics. Oncogene 23: 8105-8117.

42. Mufti, A. R., E. Burstein, R. A. Csomos, P. C. Graf, J. C. Wilkinson, R. D. Dick, M. Challa, J. K. Son, S. B. Bratton, G. L. Su, G. J. Brewer, U. Jakob, and C. S. Duckett. 2006. XIAP is a copper binding protein deregulated in Wilson's disease and other copper toxicosis disorders. Mol. Cell 21:775-785.

43. Nesvizhskii, A. I., A. Keller, E. Kolker, and R. Aebersold. 2003. A statistical model for identifying proteins by tandem mass spectrometry. Anal. Chem. 75:4646-4658

44. Ooi, C. E., E. Rabinovich, A. Dancis, J. S. Bonifacino, and R. D. Klausner. 1996. Copper-dependent degradation of the Saccharomyces cerevisiae plasma membrane copper transporter Ctr1p in the apparent absence of endocytosis. EMBO J. 15:3515-3523.

45. O'Riordan, M. X. D., L. D. Bauler, F. L. Scott, and C. S. Duckett. 2008. Inhibitor of apoptosis proteins in eukaryotic evolution and development: a model of thematic conservation. Dev. Cell 15:497-508

46. Petris, M. J., K. Smith, J. Lee, and D. J. Thiele. 2003. Copper-stimulated endocytosis and degradation of the human copper transporter, hCtr1. J. Biol. Chem. 278:9639-9646.

47. Prohaska, J. R., M. Broderius, and B. Brokate. 2003. Metallochaperone for $\mathrm{Cu}, \mathrm{Zn}$-superoxide dismutase (CCS) protein but not mRNA is higher in organs from copper-deficient mice and rats. Arch. Biochem. Biophys. 417: $227-234$.

48. Qin, X. F., D. S. An, I. S. Chen, and D. Baltimore. 2003. Inhibiting HIV-1 infection in human T cells by lentiviral-mediated delivery of small interfering RNA against CCR5. Proc. Natl. Acad. Sci. U. S. A. 100:183-188.

49. Rae, T. D., P. J. Schmidt, R. A. Pufahl, V. C. Culotta, and T. V. O'Halloran. 1999. Undetectable intracellular free copper: the requirement of a copper chaperone for superoxide dismutase. Science 284:805-808.

50. Rees, E. M., and D. J. Thiele. 2004. From aging to virulence: forging connections through the study of copper homeostasis in eukaryotic microorganisms. Curr. Opin. Microbiol. 7:175-184.

51. Rumble, J. M., M. J. Bertrand, R. A. Csomos, C. W. Wright, L. Albert, T. W. Mak, P. A. Barker, and C. S. Duckett. 2008. Apoptotic sensitivity of murine IAP-deficient cells. Biochem. J. 415:21-25.

52. Schmidt, P. J., T. D. Rae, R. A. Pufahl, T. Hamma, J. Strain, T. V. O'Halloran, and V. C. Culotta. 1999. Multiple protein domains contribute to the action of the copper chaperone for superoxide dismutase. J. Biol. Chem. 274:23719-23725.

53. Son, M., Q. Fu, K. Puttaparthi, C. M. Matthews, and J. L. Elliott. 2009 Redox susceptibility of SOD1 mutants is associated with the differential response to CCS over-expression in vivo. Neurobiol. Dis. 34:155-162.

54. Son, M., K. Puttaparthi, H. Kawamata, B. Rajendran, P. J. Boyer, G. Manfredi, and J. L. Elliott. 2007. Overexpression of CCS in G93A-SOD1 mice 
leads to accelerated neurological deficits with severe mitochondrial pathology. Proc. Natl. Acad. Sci. U. S. A. 104:6072-6077.

55. Srinivasula, S. M., and J. D. Ashwell. 2008. IAPs: what's in a name? Mol. Cell 30:123-135.

56. Subramaniam, J. R., W. E. Lyons, J. Liu, T. B. Bartnikas, J. Rothstein, D. L. Price, D. W. Cleveland, J. D. Gitlin, and P. C. Wong. 2002. Mutant SOD1 causes motor neuron disease independent of copper chaperone-mediated copper loading. Nat. Neurosci. 5:301-307.

57. Tagwerker, C., K. Flick, M. Cui, C. Guerrero, Y. Dou, B. Auer, P. Baldi, L. Huang, and P. Kaiser. 2006. A tandem affinity tag for two-step purification under fully denaturing conditions: application in ubiquitin profiling and protein complex identification combined with in vivo cross-linking. Mol. Cell. Proteomics 5:737-748.

58. Tao, T. Y., F. Liu, L. Klomp, C. Wijmenga, and J. D. Gitlin. 2003. The copper toxicosis gene product murr1 directly interacts with the Wilson disease protein. J. Biol. Chem. 278:41593-41596.

59. Thiele, D. J. 1988. ACE1 regulates expression of the Saccharomyces cerevisiae metallothionein gene. Mol. Cell. Biol. 8:2745-2752.

60. Turski, M. L., and D. J. Thiele. 2009. New roles for copper metabolism in cell proliferation, signaling, and disease. J. Biol. Chem. 284:717-721.

61. Uren, A., M. Pakusch, C. Hawkins, K. L. Puls, and D. L. Vaux. 1996. Cloning and expression of apoptosis inhibitory protein homologs that function to inhibit apoptosis and/or bind tumor necrosis factor receptor-associated factors. Proc. Natl. Acad. Sci. U. S. A. 93:4974-4978.

62. van de Sluis, B., P. Muller, K. Duran, A. Chen, A. J. Groot, L. W. Klomp, P. P. Liu, and C. Wijmenga. 2007. Increased activity of hypoxia-inducible factor 1 is associated with early embryonic lethality in Commd1 null mice. Mol. Cell. Biol. 27:4142-4156.

63. van de Sluis, B., J. Rothuizen, P. L. Pearson, B. A. van Oost, and C. Wijmenga. 2002. Identification of a new copper metabolism gene by positional cloning in a purebred dog population. Hum. Mol. Genet. 11:165-173.

64. Wilkinson, J. C., A. S. Wilkinson, S. Galbán, R. A. Csomos, and C. S. Duckett. 2008. Apoptosis-inducing factor is a target for ubiquitination through interaction with XIAP. Mol. Cell. Biol. 28:237-247.

65. Winsauer, G., U. Resch, R. Hofer-Warbinek, Y. M. Schichl, and R. de Martin. 2008. XIAP regulates bi-phasic NF- $\mathrm{B}$ induction involving physical interaction and ubiquitination of MEKK2. Cell. Signal. 20:2107-2112.

66. Wong, P. C., D. Waggoner, J. R. Subramaniam, L. Tessarollo, T. B. Bartnikas, V. C. Culotta, D. L. Price, J. Rothstein, and J. D. Gitlin. 2000. Copper chaperone for superoxide dismutase is essential to activate mammalian $\mathrm{Cu} / \mathrm{Zn}$ superoxide dismutase. Proc. Natl. Acad. Sci. U. S. A. 97:2886-2891.

67. Yang, Y., S. Fang, J. P. Jensen, A. M. Weissman, and J. D. Ashwell. 2000 Ubiquitin protein ligase activity of IAPs and their degradation in proteasomes in response to apoptotic stimuli. Science 288:874-877. 